
\title{
28 Research Square \\ Differentially expressed IGF2BP2 in ovarian disorders: strongly associates with alternative splicing regulation in human granulosa cells
}

\section{Feiyan Zhao}

Capital Medical University Beijing Obstetrics and Gynecology Hospital

Qin Wang

Capital Medical University Beijing Obstetrics and Gynecology Hospital

\section{Tong Chen}

Capital Medical University Beijing Obstetrics and Gynecology Hospital

\section{Xuehan Zhao}

Capital Medical University Beijing Obstetrics and Gynecology Hospital

\section{Zhimin Xin}

Capital Medical University Beijing Obstetrics and Gynecology Hospital

Xiaokui Yang ( $\nabla$ yangxiaokui@ccmu.edu.cn )

Capital Medical University Beijing Obstetrics and Gynecology Hospital https://orcid.org/0000-00018592-9262

\section{Research Article}

Keywords: IGF2BP2, RNA-binding proteins, RIP-seq, alternative splicing, ovarian disorders

Posted Date: March 15th, 2021

DOl: https://doi.org/10.21203/rs.3.rs-285355/v1

License: (c) (1) This work is licensed under a Creative Commons Attribution 4.0 International License. Read Full License 


\section{Abstract}

Genome-wide interactions between RNA-binding proteins (RBPs) and RNA targets account for an important portion of post-transcriptional regulation. IGF2BP2 is associated with type 2 diabetes (T2D) and obesity and reportedly functions as an RBP that regulates a series of target genes by binding RNA transcripts. In this study, we detected the differential expression of IGF2BP2 in granulosa cells from women with ovarian disorders and performed RNA-seq and RIP-seq experiments in immortalized human granulosa cells (KGN cells) to evaluate global transcript levels and alternative splicing on KGN cells overexpressing IGF2BP2 versus controls. Our results show that IGF2BP2 preferentially binds to the 3'and $5^{\prime} U T R$ s of mRNAs and enriches target gene expression in KGN cells. Notably, besides the conventional GGAC motif, we found a significant enrichment of a new GAAG motif within IGF2BP2-binding regions. We demonstrate that IGF2BP2 is involved in transcription regulation and alternative splicing in genes associated with follicular development. Furthermore, IGF2BP2 partly influences the expression levels of some of these alternatively spliced genes, including MBD3 and FN1, which may lead to ovarian endocrine disorders. In conclusion, we provide a transcriptome-wide analysis that demonstrates the role played by IGF2BP2 in the regulation of gene expression, transcription and alternative splicing of a number of genes involved in the pathogenesis of ovarian endocrine diseases.

\section{Introduction}

The ovary is an important reproductive organ in females that performs a variety of functions including generating fertile oocytes, secreting reproductive hormones and maintaining estrous cycles. These are complex biological processes that require transcriptional regulation of a large number of genes [1]. Ovarian granulosa cells (GCs), which serve as functional somatic cells that surround oocytes, can interact with the developing oocyte and are therefore essential for folliculogenesis. Aberrant pathological changes of GCs may result in follicle loss and eventually lead to several ovarian endocrine disorders, such as polycystic ovary syndrome (PCOS) and premature ovarian insufficiency (POI). Amounting evidence from a variety of research studies is revealing the specific mechanisms associated with GCs regulation and their effect on follicle development. At present, RNA-binding proteins (RBPs) are gaining recognition for their importance in unveiling the mechanistic processes behind the pathogenesis of ovaries [2-5].

RBPs that bind to RNA target sequences and form ribonucleoprotein complexes play key roles in various gene functions in eukaryotic organisms. Specific interactions between RBPs and their RNA targets represent important levels of post-transcriptional regulation, including the regulation of gene expression at the level of pre-mRNA splicing and polyadenylation, mRNA stability, mRNA localization and translation. Alternative splicing (AS) is an important mechanism for controlling gene expression, which regulates tissue identity and numerous critical biological processes. Furthermore, increasing evidence indicates that RBPs are able to promote exon inclusion or skipping by binding to splicing regulatory elements [6-8]. Finally, change in the levels and activity of RBPs may lead to AS dysregulation and the production of diverse aberrant transcripts that might contribute to some diseases in humans. 
Insulin-like growth factor 2 mRNA-binding protein 2 (IGF2BP2), also known as IMP2 or VICKZ2, is a member of the IGF2 mRNA-binding protein family and encoded by the IGF2BP2 gene, which is located on chromosome 3q27 [9-11]. IGF2BP2 is associated with type 2 diabetes (T2D) and obesity, has been reported to be also involved in insulin resistance, lipid metabolism, and tumorigenesis [12,13]. Igf $2 b p 2$ is expressed in multiple organs and tissues, where it may exert a regulatory function. For example, previous studies reported that IGF2BPs (IMPs) such as IGF2BP2 are expressed in male and female gonadal cells at embryonic day 12.5 (E12.5) in mice and that IGF2BP1, IGF2BP2 and IGF2BP3 are present in resting and growing oocytes as well as in the granulosa cells of mature mouse and human ovaries [14].

It has been recently reported that IGF2BP2 is highly expressed in the granulosa cells of women with PCOS and participates in the high mobility group AT hook 2 (HMGA2)/IMP2 pathway to promote the proliferation of granulosa cells [15]. However, the comprehensive role played by IGF2BP2 in the pathogenesis of ovarian diseases remains poorly understood. In this study, we generated IGF2BP2overexpression immortalized human granulosa cells (KGN cells) and obtained IGF2BP2-regulated transcriptomes in the KGN cell line HPB-ALL by RNA sequencing (RNA-seq) in order to study the potential function of IGF2BP2 in the regulation of gene expression that is potentially linked to ovarian diseases. We also performed RNA-immunoprecipitation (IP) sequencing (RIP-seq) in KGN cells to comprehensively identify the mRNAs which are associated with IGF2BP2. Comparative transcriptome analysis revealed that IGF2BP2 binding is strongly associated with the regulation of alternative splicing in KGN cells and partly influences the expression of a variety of genes linked to the pathogenesis of ovarian endocrine disorders.

\section{Materials And Methods}

\section{Clinical samples}

A total of 30 women were enrolled in the study in the Department of Human Reproductive Medicine, Beijing Obstetrics and Gynecology Hospital from January 1, 2019 to January 31, 2021. They routinely signed informed consent after a detailed explanation about some subsequent data collection for further study. The diagnostic criteria of POI are as follows: 1) younger than 40 years of age; 2) at least 1 year of amenorrhea, 3) two or more instances in which the serum FSH level is greater than $35 \mathrm{U} / \mathrm{L}$ (ie, two analyses at intervals of $1 \mathrm{mo}$ or more), and 4) serum estradiol levels less than $20 \mathrm{pg} / \mathrm{mL}$. Women with PCOS were selected in accordance with the Rotterdam criteria [16], including oligoovulation and/or anovulation, clinical and/or biochemical signs of hyperandrogenism, and polycystic ovarian morphology by ultrasound. All women were 20 to 35 years old and with at least two serum concentrations of FSH $<10$ $\mathrm{IU} / \mathrm{L}$ and anti-Müllerian hormone $>1 \mathrm{ng} / \mathrm{mL}$ obtained at least 1 month apart. Control group included women of comparable age with regular menses and normal basic endocrine parameters and bilateral antral follicle counts (single antral follicle count $=6$ to 10) who had received IVF-ET/ICSI (in vitro fertilization-embryo transfer/intracytoplasmic sperm injection) for male and/or tubal factor infertility and who had no family history of T2D. Patients were excluded if they had a diagnosis of uterine fibroids, pelvic tuberculosis, autoimmune diseases, genital organ deformity, metabolic disorders, recurrent 
miscarriage, recurrent implantation failure, chromosomal abnormality, fertility caused by tubal factor or male factor, a history of other ovarian surgery, or received a steroid or immunosuppressant within the preceding 6 months. The study was approved by the Ethics Committee of the Beijing Obstetrics and Gynecology Hospital, Capital Medical University.

\section{Preparation of human GCs}

Human granulosa cells were isolated from follicular fluid aspirates obtained at oocyte retrieval as described by Shi et al.[17]. Briefly, follicular fluid from each patient was centrifuged at $400 \times g$ for 10 minutes, and the layers of granulosa cells with the red blood cell pellet were resuspended. After shaking at $200 \mathrm{rpm}$ for 20 minutes at $37^{\circ} \mathrm{C}$, the cell suspensions were layered on $8.0 \mathrm{~mL}$ Ficoll-Paque Plus (GE Healthcare, Pittsburgh, PA) and centrifuged at $600 \times g$ for 20 minutes. Granulosa cells at the interface were harvested and washed three times with $10 \mathrm{~mL}$ Dulbecco's modified Eagle medium (DMEM)/nutrient mixture F-12 Ham (DMEM/F-12) supplemented with 10\% fetal bovine serum, $100 \mathrm{U} / \mathrm{mL}$ penicillin, 100 $\mu \mathrm{g} / \mathrm{mL}$ streptomycin sulfate, and $1 \times$ GlutaMAX (Invitrogen, Shanghai, China). After a final centrifugation for 5 minutes at $600 \times g$, the cells were prepared for the following RNA and protein extraction.

\section{Cloning and Plasmid Construction}

Primer pairs used for Hot Fusion were designed with CE Design V1.04. Each of the primer comprises of a specific gene fragment sequence and a 17-30 bp sequence of the pIRES-hrGFP-1 1 vector. The forward $(F)$ and reverse $(R)$ sequences of the primers used were:

F-primer: agcccgggcggatccgaattcATGATGAACAAGCTTTACA TCG

R-primer: gtcatccttgtagtcctcgagCTTGCTGCGCTGTGAGGC

The pIRES-hrGFP-1a vector was digested by EcoRI and Xhol (NEB) at $37^{\circ} \mathrm{C}$ for $2 \mathrm{~h}-3 \mathrm{~h}$. After digestion, we ran the vector on a $1.0 \%$ agarose gel and purified using a Qiagen column kit. Total RNA was isolated from KGN cells with Trizol. Purified RNA was transcribed for cDNA by oligo dT primer, after which the insert fragment was synthesized by PCR amplification. We added the linearized vector (i.e. digested with EcoRI and Xhol) and the PCR insert to a PCR microtube for ligation with ClonExpress ${ }^{\circledR}$ II One Step Cloning Kit (Vazyme). The plasmids were introduced into an Escherichia coli strain by chemical transformation, and the cells were plated onto LB agar plates containing $1 \mu \mathrm{L} / \mathrm{ml}$ ampicillin and incubated overnight at $37^{\circ} \mathrm{C}$. The colonies were screened by colony PCR ( 28 cycles) with universal primers (located on the backbone vector). The insert sequence was confirmed by Sanger sequencing.

\section{Cell culture and transfections}


$\mathrm{KGN}$ cells were cultured at $37^{\circ} \mathrm{C}$ with $5 \% \mathrm{CO}_{2}$ in DMEM/F12 with $10 \%$ fetal bovine serum (FBS), 100 $\mu \mathrm{g} / \mathrm{mL}$ streptomycin, $100 \mathrm{U} / \mathrm{mL}$ penicillin. We performed plasmid transfection of $\mathrm{KGN}$ cells using Lipofectamine 2000 (Invitrogen, Carlsbad, CA, USA) according to the manufacturer's protocol. Transfected cells were harvested after $48 \mathrm{~h}$ for RT-qPCR analysis.

\section{Assessment of gene overexpression}

GAPDH (glyceraldehyde-3-phosphate dehydrogenase) was used as a control gene for assessing the effects of IGF2BP2 overexpression. cDNA synthesis was done using standard procedures and RT-qPCR was performed on a Bio-Rad S1000 with Bestar SYBR Green RT-PCR Master Mix (DBI Bioscience, Shanghai, China). A detailed information about the primers used is presented in Supplementary Table 1. The concentration of each transcript was normalized to GAPDH mRNA level using $2^{-\triangle \triangle C T}$ method (Livak and Schmittgen 2001). We performed statistical comparisons with a paired Student's t-test using the GraphPad Prism software (San Diego, CA).

\section{RNA extraction and sequencing}

The KGN cells were ground into fine powder before RNA extraction. Total RNA was treated with RQ1 DNase (Promega) to remove DNA. The quality and quantity of the purified RNA were determined by measuring the absorbance at 260nm/280nm (A260/A280) using smartspec plus (BioRad). RNA integrity was confirmed by $1.5 \%$ Agarose gel electrophoresis.

For each sample, $0.5 \mu \mathrm{g}$ of total RNA was used for RNA-seq library preparation using the KAPA Stranded mRNA-Seq Kit for Illumina ${ }^{\circledR}$ Platforms (KK8544). Polyadenylated mRNAs were purified, fragmented and converted into double strand cDNA. Following end repair and A tailing, the DNA fragments were ligated to the Diluted Roche Adaptor (KK8726). After purifying and size fractioning to $300-500 \mathrm{bps}$, the ligated products were amplified and purified, quantified and stored at $-80^{\circ} \mathrm{C}$ before sequencing. The strand marked with dUTP (the 2nd cDNA strand) was not amplified, allowing for strand-specific sequencing.

The libraries were prepared for high-throughput sequencing following the manufacturer's instructions for the Illumina NovaSeq 6000 system with 150 nt paired-end sequencing.

\section{RNA-Seq Raw Data Clean and Alignment}

We discarded raw reads containing more than 2-N bases and then trimmed the adaptors and low-quality bases from raw sequencing reads using the FASTX-Toolkit (Version 0.0.13). We have also discarded reads shorter than $16 \mathrm{nt}$. The dataset containing the clean reads was aligned to the GRch38 genome by 
tophat2 [18] allowing 4 mismatches. Uniquely mapped reads were used for gene reads number counting and FPKM calculation (fragments per kilobase of transcript per million fragments mapped) [19].

\section{Differentially Expressed Genes (DEG) analysis}

We utilized the R Bioconductor package edgeR [20] to obtain the differentially expressed genes (DEGs). We set a false discovery rate $<0.05$ and fold change $>2$ or $<0.5$ as the cut-off criteria for DEGs identification.

\section{Alternative splicing analysis}

The alternative splicing events (ASEs) and regulated alternative splicing events (RASEs) between the samples were defined and quantified using the ABLas pipeline as described previously $[21,22]$. Briefly, ABLas detected ten types of ASEs based on the splice junction reads, including exon skipping (ES), alternative $5^{\prime}$ splice site (A5SS), alternative 3' splice site (A3SS), intron retention (IR), mutually exclusive exons (MXE), mutually exclusive 5'UTRs (5pMXE), mutually exclusive 3'UTRs (3pMXE), cassette exon, A3SS \& ES and A5SS \& ES.

To assess RBP regulated ASEs, we evaluated the significance of the ratio alteration of AS events by performing a Student's t-test. Those events with a significant at P-value cutoff corresponding to a false discovery rate cutoff of $5 \%$ were considered RBP regulated ASEs.

\section{Reverse transcription qPCR validation of DEGs and AS events}

We performed quantitative reverse-transcription polymerase chain reaction (RT-qPCR) for some of the DEGs to elucidate the validity of the RNA-seq data. Information on the primers utilized is presented in Supplementary Table 1. Total RNA remaining from the RNA-seq library preparation was used for RT-qPCR. RNA was reverse transcribed into cDNA using a M-MLV Reverse Transcriptase (Vazyme). Real-time PCR was performed with the Step One Real Time PCR System using the SYBR Green PCR Reagents Kit (Yeasen). The PCR conditions were as follows: denaturing at $95^{\circ} \mathrm{C}$ for $10 \mathrm{~min}, 40$ cycles of denaturing at $95^{\circ} \mathrm{C}$ for $15 \mathrm{~s}$, annealing and extension at $60^{\circ} \mathrm{C}$ for $1 \mathrm{~min}$. PCR amplifications were performed in triplicate for each sample. The RNA expression levels of all genes were normalized against that of GAPDH.

Additionally, we performed a RT-qPCR assay in order to validate ASEs. Information on the primers utilized for detecting ASEs is shown in Supplementary Table 1. To detect alternative isoforms, we used a boundary-spanning primer for the sequence encompassing the junction of a constitutive exon and an alternative exon as well as an opposing primer in a constitutive exon. The boundary-spanning primer of 
the alternative exon was designed according to "model exon" to detect model splicing or "altered exon" to detect altered splicing.

\section{Functional enrichment analysis}

We identified the functional categories of DEGs using Gene Ontology (GO) terms and KEGG pathways with the KOBAS 2.0 server. We used a hypergeometric test and a Benjamini-Hochberg FDR correction to define the enrichment of each term.

\section{iRIP-seq library preparation and sequencing}

KGN cells were irradiated once for $400 \mathrm{~mJ} / \mathrm{cm} 2$, grinded in liquid nitrogen and lysed in ice-cold wash buffer. Cells lysis was performed in cold wash buffer ( $1 \times$ PBS, $0.1 \%$ SDS, $0.5 \%$ NP-40 and $0.5 \%$ sodium deoxycholate) supplemented with a $200 \mathrm{U} / \mathrm{mL}$ RNase inhibitor (Takara) and protease inhibitor cocktail (Roche) and incubated on ice for $30 \mathrm{~min}$. We cleared the cell lysate by centrifugation at 10,000 rpm for 10 min at $4^{\circ} \mathrm{C}$. We then added RQ I (promega, $1 \mathrm{U} / \mu \mathrm{l}$ ) to a final concentration of $1 \mathrm{U} / \mu \mathrm{l}$ and incubated in a water bath for $30 \mathrm{~min}$ at $37^{\circ} \mathrm{C}$. Immediately afterwards, we added a stop solution to the lysates in order to quench DNase. The mixture was then vibrated vigorously and centrifuged at $13,000 \times \mathrm{g}$ at $4^{\circ} \mathrm{C}$ for $20 \mathrm{~min}$ to remove cell debris. RNA digestion was then performed by MNase (Thermo Scientific, EN0181).

For immunoprecipitation, the supernatant was incubated overnight at $4{ }^{\circ} \mathrm{C}$ with $10 \mu \mathrm{g}$ IGF2BP-antibody (Proteintech, 11601-1-AP) and control IgG-antibody (CST, 2729S). The immunoprecipitates were further incubated with protein A / D Dynabeads for $2 \mathrm{~h}$ at $4{ }^{\circ} \mathrm{C}$. After applying the magnet and removing the supernatant, we sequentially washed the beads with lysis buffer, high-salt buffer ( $250 \mathrm{mM}$ Tris 7.4, 750 $\mathrm{mM} \mathrm{NaCl}, 10 \mathrm{mM}$ EDTA, 0.1\% SDS, 0.5\% NP-40 and 0.5 deoxycholate), and PNK buffer (50 mM Tris, 20 mM EGTA and 0.5\% NP-40) twice. The beads were then resuspended in Elution buffer ( $50 \mathrm{nM}$ Tris 8.0, 10 $\mathrm{mM}$ EDTA and $1 \%$ SDS) and incubated the suspension for $20 \mathrm{~min}$ in a heat block at $70^{\circ} \mathrm{C}$ to release the immunoprecipitated RBP with crosslinked RNA before vortex. The magnetic beads were removed on the separator and the supernatant was transferred to a clean $1.5 \mathrm{ml}$ microfuge tube. Proteinase $\mathrm{K}$ (Roche) was added into the $1 \%$ input (without immunoprecipitated囚and the immunoprecipitated RBP with crosslinked RNA to a final concentration of $1.2 \mathrm{mg} / \mathrm{ml}$, and incubated for $120 \mathrm{~min}$ at $55^{\circ} \mathrm{C}$. The RNA was purified with the Trizol reagent (Life technologies).

cDNA libraries were prepared with the KAPA RNA Hyper Prep Kit (KAPA, KK8541) according to the manufacturer's protocol. We prepared high-throughput sequencing libraries following the manufacturer's instructions for the Illumina NovaSeq 6000 system with 150 nt paired-end sequencing. 


\section{Data Analysis}

After aligning the reads to the human reference genome GRCH38 with TopHat 2(Kim, Pertea et al. 2013), we kept only those that mapped uniquely for downstream analysis. The "ABLIRC" strategy was used to identify the genomic regions of IGF2BP2 binding [22]. Reads showing an overlap of at least 1 bp were clustered as peaks. For each gene, we used computer simulation to randomly generate reads having the same number and sequence lengths as empirical reads in the peaks. The reads obtained were further mapped to the same genes to generate random max peak height from overlapping reads. We repeated the entire process 500 times and selected the peaks with heights greater than those of random max peaks ( $p$-value $<0.05$ ). The IP and input samples were analyzed independently via simulations, and the IP peaks overlapping with Input peaks were removed. We finally determined the IP target genes by the peaks and used the HOMER software to call the IP binding motifs [23].

\section{Functional enrichment analysis}

We identified the functional categories of genes associated with peaks (target genes) using Gene Ontology (GO) terms and KEGG pathways with the KOBAS 2.0 server [24]. We used a hypergeometric test and a Benjamini-Hochberg FDR correction to define the enrichment of each term.

\section{Results}

\section{IGF2BP2 is differentially expressed in GCs from patients with ovarian diseases}

We first determined the clinical features related to ovarian diseases in control and patient samples (Table 1). The average age of POI women is slightly older than the other two groups, while body mass index $\left(\mathrm{kg} / \mathrm{m}^{2}\right)$ of the women with PCOS was $25.7 \pm 4.97$, which was a little higher than the control group. Levels of follicle-stimulating hormone in POI group was significantly higher than that in the control group, which suggested poor ovarian functions in these women. Furthermore, antral follicle number was significantly much more in PCOS patients and less in POI patients compared to the normal women. We collected the follicular fluid and isolated GCs these women who underwent assisted reproductive technology in the reproductive center.

To investigate potential involvement of IGF2BP2 in etiology of ovarian disease, we detected the expression levels in collected samples from POI, PCOS and normal women. Interestingly, IGF2BP2 was downregulated and upregulated in both transcriptional and protein levels in POI and PCOS patients' GCs respectively (Fig. 1a, 1b), which was indicated that it might be an important regulator to modulate gene expression associated with ovarian disorders. 


\section{IGF2BP2 preferentially binds to $3^{\prime}$ and $5^{\prime}$ UTRs of mRNAs}

To better understanding the roles IGF2BP2 plays in the pathogenesis and interactions with other ovarian disease-related genes, the IGF2BP2-interacting transcripts were confirmed by RIP-seq analysis in KGN cells. In order to assess the specificity of IGF2BP2 pull-downs, we set IgG as the immunoprecipitation control. The IGF2BP2 antibody was used to immunoprecipitate IGF2BP2-RNA complexes from the total KGN cell lysates containing IGF2BP2 overexpressing plasmids. IGF2BP2 was detected by western blotting in the total KGN cell lysate while very few quantities were detected in the negative IgG controls. Similarly, we were able to find abundant IGF2BP2 quantities in the immunoprecipitate fraction of KGN IGF2BP2-overexpressing cells (Fig. 2a). We sequenced the cDNA libraries from the RNA inputs and the IGF2BP2 fraction using the Illumina NovaSeq 6000 system. After removing the adaptor sequences and filtering out low-quality reads, we obtained a total of 44098992 and 49754547 reads from each of the IGF2BP2 immunoprecipitates repetitions, and 58215977 and 60099967 reads from the two RNA input repetitions. We mapped these four groups of reads to the human GRCH38 genome using Tophat2 [18], about $57 \%$ aligned. From the mapping data, $6.3 \%$ and $4.9 \%$ of the total mapped reads were uniquely mapped in the immunoprecipitates. About $3.4 \%$ of the total mapped reads were uniquely mapped in the input groups.

We plotted the distribution of uniquely mapped IGF2BP2 reads across reference genomic regions and found that the RIP-seq reads were highly enriched in CDS and 3'UTR regions (Fig. 2b). Furthermore, intronic regions comprised a small percentage of the whole distribution (Fig. 2b), which is consistent with the previously reported regulation of mRNA stability and translation of IGF2BP2 [25,26]. Our analysis also revealed that the fractions of clean reads that mapped to the $3^{\prime}$ and $5^{\prime} U T R s$ were significantly higher in the IGF2BP2 immunoprecipitates compared to the RNA input. Interestingly, we observed more IGF2BP2binding peaks at CDS than in intronic regions (Fig. 2b). Overall, the immunoprecipitates showed that IGFBP2 mainly binds to 5'UTR, and that this was more apparent in IP1 than IP2 (Fig. 2c).

\section{GGAC and GAAG are IGF2BP2 binding motifs}

We searched for overrepresented motifs in the IGF2BP2-binding peaks using the software Homer (http://homer.salk.edu/homer/motif/index.html). The GA-rich motif was amongst the top 10 IGF2BP2binding motifs in two independent experiments (Fig. 3a). Moreover, and in agreement with previous studies [26], we confirmed that GGAC is an enriched binding motif. It has been previously reported that IGF2BPs act as a distinct family of m6A readers that are able to recognize the $G G$ (m6A) sequence to target mRNA transcripts. A further study showed that IGF2B2P2 may bind HMGA2 mRNA via a GGAC motif and that there are possible interactions within the circNSUN2/IGF2BP2/HMGA2 complex [27]. Importantly, we found a novel motif - GAAG - in the two immunoprecipitate experiments that rank eighth (IP1) and third (IP2), respectively (Fig. 3a). In terms of the RNA binding protein, we note that the GGAC motif was enriched at the 5' UTR and that the GAAG motif was enriched in the 3'UTR (Fig. 3b, 3c). Finally, our analysis on the distribution of reads within $1 \mathrm{~kb}$ up and downstream of the TSS and the TTS, 
respectively, showed that there was an enrichment of reads downstream of the start codon and upstream of the stop codon.

\section{IGF2BP2-bound genes are enriched in gene expression regulation}

In order to validate reliable IGF2BP2-bound genes, we called IGF2BP2-bound peaks using three different methods, namely ABlife, Piranha and CIMS. The number of overlapped peaks was calculated using ABlife. A total of 4674 peak clusters consistently and repeatedly overlapped in the two iRIP-seq sample replicates (Fig. 4a). We then performed GO and KEGG enrichment analysis to further examine the potential biological roles of these IGF2BP2-bound genes (Fig. 4b, 4c). The top 10 biological process GOterm enrichment demonstrate that IGF2BP2-bound genes are associated with the regulation of transcription from RNA polymerase II promoter, the actin cytoskeleton organization and the nerve growth factor signaling pathway. Analogously, enriched KEGG pathways included focal adhesion, pancreatic cancer, prostate cancer, proteoglycans, among others. Finally, Reactome analysis showed that IGF2BP2binding RNA are mainly associated with XBP1(s)-activated chaperone genes and IRE1 alpha-activated chaperones (Fig. 4d).

IGF2BP2 binds to the mRNAs of NFIC and FOSL2, two genes related to the regulation of ovarian disorders

To confirm IGF2BP2-binding mRNA from the iRIP-seq data, we identified all the peaks shared by both the bounds. RN7SL1, PTMS, NFIC, FOSL2, VASP and NR5A1 were selected by ranking the maxheight peaks in descending order. Among these potential binding genes, NFIC, FOSL2, NR5A1 and VASP were further verified by RIP-PCR validation. These genes have been previously reported to regulate other genes involved in the pathogenesis of primary ovarian insufficiency, a clinical syndrome that is defined by the loss of ovarian activity before the age of 40 years and that has potentially devastating consequences on a woman's fertility [28]. We provide a depiction of the read density landscape of these genes across the genome and show much higher peaks compared to IGF2BP2 input samples. Specifically, IGF2BP2 bound to the CDS and the 3 'UTR region of NFIC while the binding sites of NR5A1 included the CDS, intronic regions and the $3^{\prime} U T R$ (Fig. 5a, 5b).

\section{RNA-seq analysis of IGF2BP2 regulated transcriptome profile in KGN cells}

The IGF2BP2 protein levels were significantly higher in KGN cells transfected with an IGF2BP2overexpressing plasmid compared to controls (Fig. 6a). This was confirmed by RT-qPCR showing significantly higher mRNA levels of IGF2BP2 in overexpressing cells (Fig. 6b). IGF2BP2 overexpressing $(\mathrm{OE})$ and control cells were used to construct cDNA libraries for sequencing on an Illumina NovaSeq 6000 system. We trimmed the adaptor sequences and filtered the raw reads by removing low-quality reads and 
those containing 2-N bases. The clean reads were then mapped to the human genome GRCh38 using TopHat2: approximately $93.8 \%$ of the reads aligned and $87.37 \%$ were uniquely mapped in triplicates of IGF2BP2 OE. We quantified genes and transcripts to compare gene expression patterns across individuals. Effective IGF2BP2 overexpression was further confirmed by performing three repeated parallel RNA-seq experiments. There were 17,825 genes expressed in the RNA-seq data in groups overexpressing IGF2BP2 (Fig. 6C), of which 488 were upregulated and 653 were downregulated (following the criteria of $F C \geq 2$ or $\leq 0.5$ and FDR $<0.05$ ). The heat map showing the hierarchically clustered Pearson's correlation matrix of the transcript expression values (Fig. 6d), and the volcano plot displaying the significantly expressed genes that are associated with the overexpression of IGF2BP2 (Fig. 6e), illustrate the distinct transcription profiles between control and IGF2BP2-overexpressing samples (Fig. 6f). We subsequently examine the potential biological roles of the DEGs regulated by IGF2BP2 by conducted GO enrichment analysis that included three distinct ontologies, namely molecular function, cellular component and biological process. The results including the top $\mathrm{GO}$ enrichment results on IGF2BP2-mediated up- or downregulation are shown in Figure 6g. IGF2BP2-upregulated genes are mainly associated with processes involving inflammatory response, cell surface receptor signaling pathway, defense response to virus and cytokine-mediated signaling pathway. The IGF2BP2-downregulated genes are mainly involved in transcription, DNA-dependent regulation of transcription, pattern specification process and positive regulation of transcription from RNA polymerase II promoter. Analogously, the top ten KEGG pathways associated with IGF2BP2-upregulated genes include the intestinal immune network for IgA production, inflammatory bowel disease and cytokine-cytokine receptor interaction; while those that are downregulated by IGF2BP2 are associated with transcriptional misregulation in cancer and glycerolipid metabolism (Fig. 6h).

\section{DEGs regulated by IGF2BP2 overexpression were not attributed to the direct binding of IGF2BP2}

We found a total of 17 genes that tend to bind to IGF2BP2 among the 1124 DEGs, as shown in the Venn diagram (Fig.7a). However, we found no significant correlation between IGF2BP2-binding in these 17 genes and differentially expressed genes $(P>0.05)$. We then selected several relevant $D E G s$, including OASL, THEMIS2, BCHE, FAM133B and STAB2 that were enriched in the inflammatory response signal pathway and confirmed that the relative expression levels measured by RNA-seq data and RT-qPCR are generally consistent and show similar trends (Fig. 7b).

\section{IGF2BP2 could regulate alternative splicing events in KGN cells}

In order to shed light on the role played by IGF2BP2 on AS regulation, we used the uniquely mapped reads from the transcriptome sequencing data to explore IGF2BP2-dependent AS events in KGN cells. We detected $56.5 \%$ (207,635 out of 367,321$)$ annotated exons when comparing these uniquely mapped reads to the reference genome annotation, and 54,400 novel splice junctions using Tophat2. Furthermore, we 
uncovered 11,557 known ASEs in the model gene we named in the reference genome, and 29,625 novel ASEs. Based on a stringent $P$ value cut-off $(\leq 0.05)$ and changed AS ratio $\geq 0.2$, we were able to identify 340 high-confidence RASEs, of which the majority included intron retention (IR, 106 events), exon skipping (ES, 55 events), alternative 3' splice site (A3SS, 49 events), alternative 5'splice site (A5SS, 46 events), and cassette exon (CE, 38 events) (Fig. 8a). These results suggest that IGF2BP2 is able to globally regulate ASEs in KGN cells. Besides the AS changes attributed to transcription regulation, we also analyzed the expression of RASGs at a transcriptional level. There are different alternatively spliced genes between IGF2BP2-overexpressing and control samples but there was only one significantly regulated at a transcript level in RASGs (Fig. 8b). Therefore, the observed changes in AS events cannot be simply attributed a transcription up- or downregulation.

Our GO biological processes analysis suggests that the genes regulated by IGF2BP2 are highly enriched for protein gene expression, mRNA processing, RNA and mRNA metabolic processes, translation and cellular protein metabolic process (Fig. 8c). Similarly, the enriched KEGG pathways were related with protein processing in endoplasmic reticulum, legionellosis, mTOR signaling pathway, small cell lung cancer, RNA transport and spliceosome, among others. Hence, based on our functional analysis, it can be concluded that IGF2BP2 influences the expression of certain genes by regulating alternative splicing in KGN cells and that some of these genes included RPL26, HSPA8, RPL17, EIF4G1, PSMC5 and U2AF1 which were associated with ovarian diseases in previous studies [29-44].

\section{Validation of ASEs by IGF2BP2 overexpression in KGN cells}

A small part (67/235) of the IGF2BP2-regulated alternatively spliced genes overlapped the the set of genes that bind to IGF2BP2 (Fig. 9a), which suggests that IGF2BP2 binding might affect some alternative splicing events by binding to certain RNA targets. To further explore these alternative splicing genes directly regulated by IGF2BP2, we performed additional GO and KEGG pathway enrichment analyzes. In this case, the top hits obtained were transcription from RNA polymerase II promoter, blood coagulation, apoptotic process and gene expression (GO-terms), and Resin secretion, mRNA surveillance, glycosphingolipid biosynthesis-ganglio series (KEGG pathways) (Fig. 9b and 9c, respectively). Among these 67 IGF2BP2-regulated genes, we validated four splicing events affecting MBD3, FN1, TFDP1 and MKNK2 by PCR (see Supplementary Table 1 for primer information), which were confirmed to be 5pMXE, ES, A3SS and A3SS, respectively (Fig. 9d, 9e and S2). These results are consistent with the previous RNA-seq results. After IGF2BP2-overexpression, the ratio of splicing patterns was significantly higher in MBD3, FN1 and TFDP1 but lower in MKNK2. These results further suggest that IGF2BP2 binds to different genes and plays an important regulatory role in their AS in KGN cells.

\section{Discussion}

IGF2BP2 is highly expressed in the gonads, oocytes and embryos in both mice and in humans $[14,10]$. The gene belongs to a conserved family of RNA-binding proteins containing six characteristic RNA 
binding modules, including two N-terminal RNA recognition motifs (RRM1 and RRM2) and four C-terminal hnRNP K-homology ( $\mathrm{KH} 1$ to $\mathrm{KH} 4$ ) domains that control its RNA-binding specificity [45,9]. The expression of IGF2BP2 is widely distributed in E12.5 mouse embryos [12] and vital for RNA processing - its mRNA transcripts were detected at the 2-cell-stage during mice embryogenesis. It is likely that IGF2BP2 is associated with both energy expenditure and life span in different pathological conditions [46]. Accordingly, a large number of diseases, including diabetes, acute myelocytic leukemia [47] and colorectal carcinoma [48], may be partly attributed to the abnormal expression and dysregulation of IGF2BP2. In a previous study, IGF2BP2 was highly expressed in the granulosa cells of women with PCOS and it was possible to demonstrate that it serves as an HMGA2 target gene and can promote the proliferation of granulosa cells by the HMGA2/IGF2BP2 pathway [49]. However, despite the association between IGF2BP2 and ovarian endocrine disorders, there are previously proposed unknown regulatory mechanisms, and it remains unclear whether IGF2BP2 can regulate alternative splicing in the ovary. In the present study, we performed RNA-seq and RIP to explore whether IGF2BP2 could bind to genes associated with ovarian diseases and was able to regulate alternative splicing events.

We analyzed the binding profile of IGF2BP2 in KGN cells via RNA-seq and found that IGF2BP2 preferentially binds to the $3^{\prime}$ and $5^{\prime}$ UTRs of mRNAs (Fig. 2). In general, we confirmed the GGAC motif that was previously reported as a common binding site recognizing the $G G(m 6 A)$ sequence to target mRNA transcripts and which is involved in interactions within the circNSUN2/IGF2BP2/HMGA2 complex. More importantly, we also found a novel GAAG motif in the two immunoprecipitate experiments. We demonstrated that GGAC's binding sites were enriched at the 5' UTRs while the GAAG motif was enriched at the $3^{\prime}$ UTRs. The recognition of both 5' and 3' splice sites in pre-mRNA was an essential event in the alternative splicing decision.

Furthermore, GO and KEGG analyzes on the RIP-seq data showed that the genes binding to IGF2BP2 mainly function as regulators of gene expression, which reveals an important role in the crosstalk between gene networks. Among the IGF2BP2-binding mRNA, we found four candidates associated with ovarian insufficiency (POI): NFIC, FOSL2, NR5A1 and VASP, as demonstrated by previous studies. For example, a mutation in NR5A1 was identified in women $\mathrm{POI}[50,51,7,52]$. This mutation might result in an impaired transcriptional activation of the Amh, Inhibin-a, Cyp11a1 and Cyp19a1 genes, all of which are essential for normal ovarian function [53]. However, it is still unclear if the mutations in NR5A1 are rare and whether they contribute to pathogenicity of POI. Furthermore, FOXL2 exclusively heterodimerizes with Jun members to form an activator protein-1(AP-1) complex, thereby participating in various cellular processes including proliferation, differentiation, and transformation [54]. It has been shown that gonadotropin upregulates FOSL2 and JUNB in granulosa cells and influences the phosphorylation of the estrogen receptor beta (ESR2), which play an essential role in the onset of puberty, gonadal development and ovulation [55]. Finally, the genes NFIC and VASP have also been associated with some ovarian disorders in female reproduction [56-58].

We unbiasedly analyzed the DEGs that show evidence of being regulated by IGF2BP2 from the RNA-seq data. Functional enrichment analyzes demonstrated that these DEGs are enriched in multiple GO-terms 
and KEGG pathways. These include inflammatory response, defense response to virus and cytokinemediated signaling pathway, all of which are concordant with the reported IGF2BP2 function in regulating inflammatory environments and genomic stability in human vascular endothelial cells and hepatocytes [59]. IGF2BP2-regulated genes were also enriched in intestinal immune network for IgA production, inflammatory bowel disease and cytokine-cytokine receptor interaction.

Among the 1124 DEGs obtained from the RNA-seq data, 17 (or their antisense RNA) have IGF2BP2 binding sites (Fig. 7). Thus, it is possible that these are the only genes which might be regulated through direct binding to IGF2BP2 despite an observed lack of significant correlation between IGF2BP2-binding and regulation. The selected genes showing an association with the inflammatory response signal pathway were confirmed by qPCR, further validating the analysis above.

Notably, the majority of alternatively spliced genes that are regulated by IGF2BP2 involved gene expression without changes in transcription levels. Therefore, we hypothesize that IGF2BP2 binds to mRNA to act as a regulator of gene expression and plays an important role in the regulation of alternative splicing events. Moreover, the AS genes that are also IGF2BP2 binding targets were mainly involved in gene expression which is in line with the findings of a GO analysis comparing RASE between IGF2BP2overexpressing and control groups. The IGF2BP2-regulated AS genes in these biological processes include MBD3, FN1, TFDP1 and MKNK2, all of which were validated by RT-qPCR. We were also able to demonstrate that, after overexpressing of IGF2BP2 in KGN cells, the mRNA expression ratios of MBD3, FN1 and TFDP1 were higher, while that of MKNK2 was lower. MBD3, a member of the nucleosome remodeling deacetylase complex, is a maternal protein that is essential for the protection of imprinting control regions against DNA demethylation after fertilization and for maintaining DNA methylation of the $\mathrm{H} 19$ paternal allele during preimplantation $[60,61]$. FN1 is a glycoprotein that is present on the cell surface, in extracellular fluids and connective tissues, and a component of the basement membrane in the ovarian follicle. Fibronectin is a secretion product that is associated with granulosa cell adhesion and migration in rats $[62,63]$. The production of fibronectin is indispensable in the early stages of follicular development and tends to rise during follicle maturation [64]. Previous studies have shown that fibronectin appears during the early luteal phase and then decreases in murine granulosa cells [65], and that it might participate in the regulation of cell proliferation [66].

\section{Conclusion}

In this study, we indicated that IGF2BP2 might be involved in the regulation of transcription and alternative splicing of a series of genes associated with follicular development in human granulosa cell lines. Moreover, we show that IGF2BP2 partly influences the expression of some target alternative splicing genes, and that this may lead to the pathogenesis of ovarian disorders. However, the detailed mechanisms of these alternative splicing events and downstream regulatory patterns still require further investigation.

\section{Abbreviations}


PCOS - Polycystic ovary syndrome

POI - Premature ovarian insufficiency

RBPs - RNA-binding proteins

AS - Alternative splicing

IGF2BP2 - Insulin-like growth factor 2 mRNA-binding protein 2

T2D - Type 2 diabetes

HMGA2 - High mobility group AT hook 2

RNA-seq - RNA sequencing

RIP-seq - RNA-immunoprecipitation sequencing

FPKM - Fragments per kilobase of transcript per million

DEG - Differentially Expressed Genes

ASEs - Alternative splicing events

RASEs - Regulated alternative splicing events

ES - Exon skipping

A5SS - Alternative 5' splice site

A3SS - Alternative 3'splice site

IR - Intron retention

MXE - Mutually exclusive exons

5pMXE - Mutually exclusive 5'UTRs

3pMXE - Mutually exclusive 3'UTRs

RT-qPCR - Quantitative reverse-transcription polymerase chain reaction

TSS - Transcription start sites

TTS - Transcription termination sites

RN7SL1 - RNA 7SL cytoplasmic 1 
PTMS - Parathymosin

NF1C - Nuclear factor 1 C-type

FOSL2 - Fos-related antigen 2

VASP - Vasodilator-stimulated phosphoprotein

NR5A1 - Nuclear receptor subfamily 5 group A member 1

OASL - 2',5'-oligoadenylate synthetase-like gene

THEMIS2 - Thymocyte selection associated family member 2

BCHE - Butyrylcholinesterase

FAM133B - Family with sequence similarity 133 member B

STAB2 - Stabilin 2

RPL26 - Ribosomal protein L26

HSPA8 - Heat shock protein family A (Hsp70) member 8

RPL17 - Ribosomal protein L17

EIF4G1 - Eukaryotic translation initiation factor 4 gamma 1

PSMC5 - Proteasome 26S subunit, ATPase 5

U2AF1 - U2 small nuclear RNA auxiliary factor 1

MBD3 - Methyl-CpG binding domain protein 3

FN1 - Fibronectin 1

TFDP1 - Transcription factor Dp-1

MKNK2 - MAPK interacting serine/threonine kinase 2

RRM - RNA recognition motifs

$\mathrm{AMH}$ - Anti-Mullerian hormone

Inhibin-a - Inhibin subunit beta A

Cyp11a1 - Cytochrome P450 family 11 subfamily A member 1 
Cyp19a1 - Cytochrome P450 family 19 subfamily A member 1

AP-1 - Activator protein-1

ESR2 - Estrogen receptor beta

\section{Declarations}

\section{Funding}

This study was supported by grants from National Natural Science Foundation of China, Code: 81871133; National Natural Science Foundation of China, Code: 81471431; Beijing Hospitals Authority' Ascent Plan, Code: DFL20191401.

\section{Conflicts of interest/Competing interests}

The authors wish to declare that they have no conflict of interest.

\section{Availability of data and material}

The RNA-seq data produced in this work will be deposited in NCBI's Gene Expression Omnibus and can be accessed through GEO series accession.

\section{Code availability}

edgeR; KOBAS 2.0 server; HOMER software.

\section{Authors' contributions}

Feiyan Zhao performed most of the experiments. Qing Wang, Tong Chen and Xuehan Zhao performed and analyzed cytological identification experiments. Xiaokui Yang and Zhimin Xin designed the experiments and supervised the project. Feiyan Zhao wrote the manuscript with input from all co-authors.

\section{Ethics approval}

The authors wish to confirm the originality of this work and that is has neither been published nor is it under consideration for publication elsewhere. Ethical approval was obtained from the Ethics Committee of the Beijing Obstetrics and Gynecology Hospital, Capital Medical University.

\section{Consent to participate}

Informed consent was obtained from all individual participants included in the study.

\section{Consent for publication}

Patients signed informed consent regarding publishing their data. 


\section{Acknowledgments}

We thank Dr. Lan Yu, Dr. Rui Song, Ms Li Wang, Ms Wei Li, Ms Yuan Yuan, Dr. Qiaoyuan Chen and Ms Xiaojie Wang for collection of follicular fluid samples. We are very grateful to the members of ABLife Inc. for their technical contributions to data analysis and helpful discussions.

\section{References}

1. Tang L-T, Ran X-Q, Mao N, Zhang F-P, Niu X, Ruan Y-Q, Yi F-L, Li S, Wang J-F (2018) Analysis of alternative splicing events by RNA sequencing in the ovaries of Xiang pig at estrous and diestrous. Theriogenology 119:60-68. doi:10.1016/j.theriogenology.2018.06.022

2. Vilariño-García T, Pérez-Pérez A, Santamaría-López E, Prados N, Fernández-Sánchez M, SánchezMargalet V (2020) Sam68 mediates leptin signaling and action in human granulosa cells: possible role in leptin resistance in PCOS. Endocr Connect 9 (6):479-488. doi:10.1530/EC-20-0062

3. Bansal P, Madlung J, Schaaf K, Macek B, Bono F (2020) An Interaction Network of RNA-Binding Proteins Involved in Oogenesis. Mol Cell Proteomics 19 (9):1485-1502. doi:10.1074/mcp.RA119.001912

4. Kato Y, Iwamori T, Ninomiya Y, Kohda T, Miyashita J, Sato M, Saga Y (2019) ELAVL2-directed RNA regulatory network drives the formation of quiescent primordial follicles. EMBO Rep 20 (12):e48251. doi:10.15252/embr.201948251

5. Sutherland JM, Sobinoff AP, Gunter KM, Fraser BA, Pye V, Bernstein IR, Boon E, Siddall NA, De Andres LI, Hime GR, Holt JE, Graf T, McLaughlin EA (2015) Knockout of RNA Binding Protein MSI2 Impairs Follicle Development in the Mouse Ovary: Characterization of MSI1 and MSI2 during Folliculogenesis. Biomolecules 5 (3):1228-1244. doi:10.3390/biom5031228

6. Soucek P, Reblova K, Kramarek M, Radova L, Grymova T, Hujova P, Kovacova T, Lexa M, Grodecka L, Freiberger T (2019) High-throughput analysis revealed mutations' diverging effects on SMN1 exon 7 splicing. RNA Biol 16 (10):1364-1376. doi:10.1080/15476286.2019.1630796

7. Wang E, Lu SX, Pastore A, Chen X, Imig J, Chun-Wei Lee S, Hockemeyer K, Ghebrechristos YE, Yoshimi A, Inoue D, Ki M, Cho H, Bitner L, Kloetgen A, Lin KT, Uehara T, Owa T, Tibes R, Krainer AR, Abdel-Wahab O, Aifantis I (2019) Targeting an RNA-Binding Protein Network in Acute Myeloid Leukemia. Cancer Cell 35 (3):369-384 e367. doi:10.1016/j.ccell.2019.01.010

8. Zhu Y, Deng H, Chen X, Li H, Yang C, Li S, Pan X, Tian S, Feng S, Tan X, Matsuo M, Zhang Z (2019) Skipping of an exon with a nonsense mutation in the DMD gene is induced by the conversion of a splicing enhancer to a splicing silencer. Hum Genet 138 (7):771-785. doi:10.1007/s00439-01902036-2

9. Christiansen J, Kolte AM, Hansen T, Nielsen FC (2009) IGF2 mRNA-binding protein 2: biological function and putative role in type 2 diabetes. J Mol Endocrinol 43 (5):187-195. doi:10.1677/JME-090016 
10. Nielsen J, Christiansen J, Lykke-Andersen J, Johnsen AH, Wewer UM, Nielsen FC (1999) A family of insulin-like growth factor II mRNA-binding proteins represses translation in late development. Mol Cell Biol 19 (2):1262-1270. doi:10.1128/mcb.19.2.1262

11. Xu Y, Wang L, He J, Bi Y, Li M, Wang T, Wang L, Jiang Y, Dai M, Lu J, Xu M, Li Y, Hu N, Li J, Mi S, Chen CS, Li G, Mu Y, Zhao J, Kong L, Chen J, Lai S, Wang W, Zhao W, Ning G, China Noncommunicable Disease Surveillance G (2013) Prevalence and control of diabetes in Chinese adults. JAMA 310 (9):948-959. doi:10.1001/jama.2013.168118

12. Cao J, Mu Q, Huang H (2018) The Roles of Insulin-Like Growth Factor 2 mRNA-Binding Protein 2 in Cancer and Cancer Stem Cells. Stem Cells Int 2018:4217259. doi:10.1155/2018/4217259

13. Ruchat S-M, Elks CE, Loos RJF, Vohl M-C, Weisnagel SJ, Rankinen T, Bouchard C, Pérusse L (2009) Association between insulin secretion, insulin sensitivity and type 2 diabetes susceptibility variants identified in genome-wide association studies. Acta Diabetol 46 (3):217-226. doi:10.1007/s00592008-0080-5

14. Hammer NA, Hansen T, Byskov AG, Rajpert-De Meyts E, Grondahl ML, Bredkjaer HE, Wewer UM, Christiansen J, Nielsen FC (2005) Expression of IGF-II mRNA-binding proteins (IMPs) in gonads and testicular cancer. Reproduction 130 (2):203-212. doi:10.1530/rep.1.00664

15. Li M, Zhao H, Zhao S-G, Wei D-M, Zhao Y-R, Huang T, Muhammad T, Yan L, Gao F, Li L, Lu G, Chan WY, Leung PCK, Dunaif A, Liu H-B, Chen Z-J (2019) The HMGA2-IMP2 Pathway Promotes Granulosa Cell Proliferation in Polycystic Ovary Syndrome. J Clin Endocrinol Metab 104 (4):1049-1059. doi:10.1210/jc.2018-00544

16. Rotterdam EA-SPcwg (2004) Revised 2003 consensus on diagnostic criteria and long-term health risks related to polycystic ovary syndrome (PCOS). Hum Reprod 19 (1):41-47. doi:10.1093/humrep/deh098

17. Shi FT, Cheung AP, Klausen C, Huang HF, Leung PC (2010) Growth differentiation factor 9 reverses activin A suppression of steroidogenic acute regulatory protein expression and progesterone production in human granulosa-lutein cells. J Clin Endocrinol Metab 95 (10):E172-180. doi:10.1210/jc.2010-0477

18. Kim D, Pertea G, Trapnell C, Pimentel H, Kelley R, Salzberg SL (2013) TopHat2: accurate alignment of transcriptomes in the presence of insertions, deletions and gene fusions. Genome Biol 14 (4):R36. doi:10.1186/gb-2013-14-4-r36

19. Trapnell C, Williams BA, Pertea G, Mortazavi A, Kwan G, van Baren MJ, Salzberg SL, Wold BJ, Pachter L (2010) Transcript assembly and quantification by RNA-Seq reveals unannotated transcripts and isoform switching during cell differentiation. Nat Biotechnol 28 (5):511-515. doi:10.1038/nbt.1621

20. Robinson MD, McCarthy DJ, Smyth GK (2010) edgeR: a Bioconductor package for differential expression analysis of digital gene expression data. Bioinformatics 26 (1):139-140. doi:10.1093/bioinformatics/btp616

21. Jin L, Li G, Yu D, Huang W, Cheng C, Liao S, Wu Q, Zhang Y (2017) Transcriptome analysis reveals the complexity of alternative splicing regulation in the fungus Verticillium dahliae. BMC Genomics 18 
(1):130. doi:10.1186/s12864-017-3507-y

22. Xia H, Chen D, Wu Q, Wu G, Zhou Y, Zhang Y, Zhang L (2017) CELF1 preferentially binds to exonintron boundary and regulates alternative splicing in HeLa cells. Biochim Biophys Acta Gene Regul Mech 1860 (9):911-921. doi:10.1016/j.bbagrm.2017.07.004

23. Heinz S, Benner C, Spann N, Bertolino E, Lin YC, Laslo P, Cheng JX, Murre C, Singh H, Glass CK (2010) Simple combinations of lineage-determining transcription factors prime cis-regulatory elements required for macrophage and B cell identities. Mol Cell 38 (4):576-589.

doi:10.1016/j.molcel.2010.05.004

24. Xie C, Mao X, Huang J, Ding Y, Wu J, Dong S, Kong L, Gao G, Li C-Y, Wei L (2011) KOBAS 2.0: a web server for annotation and identification of enriched pathways and diseases. Nucleic Acids Res 39 (Web Server issue):W316-W322. doi:10.1093/nar/gkr483

25. Biswas J, Patel VL, Bhaskar V, Chao JA, Singer RH, Eliscovich C (2019) The structural basis for RNA selectivity by the IMP family of RNA-binding proteins. Nat Commun 10 (1):4440. doi:10.1038/s41467-019-12193-7

26. Huang H, Weng H, Sun W, Qin X, Shi H, Wu H, Zhao BS, Mesquita A, Liu C, Yuan CL, Hu Y-C, Hüttelmaier S, Skibbe JR, Su R, Deng X, Dong L, Sun M, Li C, Nachtergaele S, Wang Y, Hu C, Ferchen K, Greis KD, Jiang X, Wei M, Qu L, Guan J-L, He C, Yang J, Chen J (2018) Recognition of RNA Nmethyladenosine by IGF2BP proteins enhances mRNA stability and translation. Nat Cell Biol 20 (3):285-295. doi:10.1038/s41556-018-0045-z

27. He R-Z, Jiang J, Luo D-X (2019) M6A modification of circNSUN2 promotes colorectal liver metastasis. Genes \& Diseases. doi:https://doi.org/10.1016/j.gendis.2019.12.002

28. Domniz N, Meirow D (2019) Premature ovarian insufficiency and autoimmune diseases. Best Pract Res Clin Obstet Gynaecol 60:42-55. doi:10.1016/j.bpobgyn.2019.07.008

29. Sotomaru Y, Kawase Y, Ueda T, Obata Y, Suzuki H, Domeki I, Hatada I, Kono T (2001) Disruption of imprinted expression of U2afbp-rs/U2af1-rs1 gene in mouse parthenogenetic fetuses. J Biol Chem 276 (28):26694-26698

30. Shibata H, Ueda T, Kamiya M, Yoshiki A, Kusakabe M, Plass C, Held WA, Sunahara S, Katsuki M, Muramatsu M, Hayashizaki Y (1997) An oocyte-specific methylation imprint center in the mouse U2afbp-rs/U2af1-rs1 gene marks the establishment of allele-specific methylation during preimplantation development. Genomics 44 (2):171-178

31. Sunahara S, Nakamura K, Nakao K, Gondo Y, Nagata Y, Katsuki M (2000) The oocyte-specific methylated region of the U2afbp-rs/U2af1-rs1 gene is dispensable for its imprinted methylation. Biochem Biophys Res Commun 268 (2):590-595

32. Haouzi D, Boumela I, Chebli K, Hamamah S (2018) Global, Survival, and Apoptotic Transcriptome during Mouse and Human Early Embryonic Development. Biomed Res Int 2018:5895628. doi:10.1155/2018/5895628

33. de Broucker A, Semaille P, Cailliau K, Martoriati A, Comptdaer T, Bodart J-F, Destée A, Chartier-Harlin M-C (2015) Xenopus laevis as a Model to Identify Translation Impairment. J Vis Exp (103). 
doi:10.3791/52724

34. Li L, Luo Q, Xie Z, Li G, Mao C, Liu Y, Wen X, Yin N, Cao J, Wang J, Li L, Yu J, Wang F, Yi P (2016) Characterization of the Expression of the RNA Binding Protein eIF4G1 and Its Clinicopathological Correlation with Serous Ovarian Cancer. PLoS One 11 (9):e0163447. doi:10.1371/journal.pone.0163447

35. Bermúdez MG, Wells D, Malter H, Munné S, Cohen J, Steuerwald NM (2004) Expression profiles of individual human oocytes using microarray technology. Reprod Biomed Online 8 (3):325-337

36. Peaston AE, Evsikov AV, Graber JH, de Vries WN, Holbrook AE, Solter D, Knowles BB (2004) Retrotransposons regulate host genes in mouse oocytes and preimplantation embryos. Dev Cell 7 (4):597-606. doi:10.1016/j.devcel.2004.09.004

37. Shan N, Zhou W, Zhang S, Zhang Y (2016) Identification of HSPA8 as a candidate biomarker for endometrial carcinoma by using iTRAQ-based proteomic analysis. Onco Targets Ther 9:2169-2179. doi:10.2147/OTT.S97983

38. Alvarez-Rodríguez M, Alvarez M, Borragan S, Martinez-Pastor F, Holt WV, Fazeli A, de Paz P, Anel L (2013) The addition of heat shock protein HSPA8 to cryoprotective media improves the survival of brown bear (Ursus arctos) spermatozoa during chilling and after cryopreservation. Theriogenology 79 (3):541-550. doi:10.1016/j.theriogenology.2012.11.006

39. Anderson RA, Sciorio R, Kinnell H, Bayne RAL, Thong KJ, de Sousa PA, Pickering S (2009) Cumulus gene expression as a predictor of human oocyte fertilisation, embryo development and competence to establish a pregnancy. Reproduction (Cambridge, England) 138 (4):629-637. doi:10.1530/REP-090144

40. Elliott RMA, Lloyd RE, Fazeli A, Sostaric E, Georgiou AS, Satake N, Watson PF, Holt WV (2009) Effects of HSPA8, an evolutionarily conserved oviductal protein, on boar and bull spermatozoa.

Reproduction (Cambridge, England) 137 (2):191-203. doi:10.1530/REP-08-0298

41. Reading BJ, Chapman RW, Schaff JE, Scholl EH, Opperman CH, Sullivan CV (2012) An ovary transcriptome for all maturational stages of the striped bass (Morone saxatilis), a highly advanced perciform fish. BMC Res Notes 5:111. doi:10.1186/1756-0500-5-111

42. Diao F-Y, Xu M, Hu Y, Li J, Xu Z, Lin M, Wang L, Zhou Y, Zhou Z, Liu J, Sha J (2004) The molecular characteristics of polycystic ovary syndrome (PCOS) ovary defined by human ovary cDNA microarray. Journal of molecular endocrinology 33 (1):59-72

43. Nilsson E, Larsen G, Manikkam M, Guerrero-Bosagna C, Savenkova MI, Skinner MK (2012) Environmentally induced epigenetic transgenerational inheritance of ovarian disease. PLoS One 7 (5):e36129. doi:10.1371/journal.pone.0036129

44. Estep AS, Sanscrainte ND, Becnel JJ (2016) DsRNA-mediated targeting of ribosomal transcripts RPS6 and RPL26 induces long-lasting and significant reductions in fecundity of the vector Aedes aegypti. J Insect Physiol 90:17-26. doi:10.1016/j.jinsphys.2016.05.001

45. Saxena R, Voight BF, Lyssenko V, Burtt NP, de Bakker PIW, Chen H, Roix JJ, Kathiresan S, Hirschhorn JN, Daly MJ, Hughes TE, Groop L, Altshuler D, Almgren P, Florez JC, Meyer J, Ardlie K, Bengtsson 
Boström K, Isomaa B, Lettre G, Lindblad U, Lyon HN, Melander O, Newton-Cheh C, Nilsson P, OrhoMelander M, Råstam L, Speliotes EK, Taskinen M-R, Tuomi T, Guiducci C, Berglund A, Carlson J, Gianniny L, Hackett R, Hall L, Holmkvist J, Laurila E, Sjögren M, Sterner M, Surti A, Svensson M, Svensson M, Tewhey R, Blumenstiel B, Parkin M, Defelice M, Barry R, Brodeur W, Camarata J, Chia N, Fava M, Gibbons J, Handsaker B, Healy C, Nguyen K, Gates C, Sougnez C, Gage D, Nizzari M, Gabriel SB, Chirn G-W, Ma Q, Parikh H, Richardson D, Ricke D, Purcell S (2007) Genome-wide association analysis identifies loci for type 2 diabetes and triglyceride levels. Science 316 (5829):1331-1336

46. Ikemoto A, Sato DX, Makino T, Kawata M (2020) Genetic factors for short life span associated with evolution of the loss of flight ability. Ecol Evol 10 (12):6020-6029. doi:10.1002/ece3.6342

47. He X, Li W, Liang X, Zhu X, Zhang L, Huang Y, Yu T, Li S, Chen Z (2018) IGF2BP2 Overexpression Indicates Poor Survival in Patients with Acute Myelocytic Leukemia. Cell Physiol Biochem 51 (4):1945-1956. doi:10.1159/000495719

48. Li T, Hu PS, Zuo Z, Lin JF, Li X, Wu QN, Chen ZH, Zeng ZL, Wang F, Zheng J, Chen D, Li B, Kang TB, Xie D, Lin D, Ju HQ, Xu RH (2019) METTL3 facilitates tumor progression via an m(6)A-IGF2BP2dependent mechanism in colorectal carcinoma. Mol Cancer 18 (1):112. doi:10.1186/s12943-0191038-7

49. Li M, Zhao H, Zhao SG, Wei DM, Zhao YR, Huang T, Muhammad T, Yan L, Gao F, Li L, Lu G, Chan WY, Leung PCK, Dunaif A, Liu HB, Chen ZJ (2019) The HMGA2-IMP2 Pathway Promotes Granulosa Cell Proliferation in Polycystic Ovary Syndrome. J Clin Endocrinol Metab 104 (4):1049-1059. doi:10.1210/jc.2018-00544

50. Lourenço D, Brauner R, Lin L, De Perdigo A, Weryha G, Muresan M, Boudjenah R, Guerra-Junior G, Maciel-Guerra AT, Achermann JC, McElreavey K, Bashamboo A (2009) Mutations in NR5A1 associated with ovarian insufficiency. N Engl J Med 360 (12):1200-1210. doi:10.1056/NEJMoa0806228

51. Harrison SM, Campbell IM, Keays M, Granberg CF, Villanueva C, Tannin G, Zinn AR, Castrillon DH, Shaw CA, Stankiewicz P, Baker LA (2013) Screening and familial characterization of copy-number variations in NR5A1 in 46,XY disorders of sex development and premature ovarian failure. Am J Med Genet A 161A (10):2487-2494. doi:10.1002/ajmg.a.36084

52. Janse F, de With LM, Duran KJ, Kloosterman WP, Goverde AJ, Lambalk CB, Laven JS, Fauser BC, Giltay JC, Dutch Primary Ovarian Insufficiency C (2012) Limited contribution of NR5A1 (SF-1) mutations in women with primary ovarian insufficiency (POI). Fertil Steril 97 (1):141-146 e142. doi:10.1016/j.fertnstert.2011.10.032

53. Jiao X, Qin Y, Li G, Zhao S, You L, Ma J, Simpson JL, Chen ZJ (2013) Novel NR5A1 missense mutation in premature ovarian failure: detection in han chinese indicates causation in different ethnic groups. PLoS One 8 (9):e74759. doi:10.1371/journal.pone.0074759

54. Ren W, Yang L, Deng T, Wu C, Li Y, Wu J, Huang Z, Du F, Guo L (2019) Calcitonin gene-related peptide regulates FOSL2 expression and cell proliferation of BMSCs via mmu_circRNA_003795. Mol Med Rep 19 (5):3732-3742. doi:10.3892/mmr.2019.10038 
55. Chakravarthi VP, Khristi V, Ghosh S, Yerrathota S, Dai E, Roby KF, Wolfe MW, Rumi MAK (2018) ESR2 Is Essential for Gonadotropin-Induced Kiss1 Expression in Granulosa Cells. Endocrinology 159 (11):3860-3873. doi:10.1210/en.2018-00608

56. Schiewek J, Schumacher U, Lange T, Joosse SA, Wikman H, Pantel K, Mikhaylova M, Kneussel M, Linder S, Schmalfeldt B, Oliveira-Ferrer L, Windhorst S (2018) Clinical relevance of cytoskeleton associated proteins for ovarian cancer. J Cancer Res Clin Oncol 144 (11):2195-2205. doi:10.1007/s00432-018-2710-9

57. Li J, Ren L, Yang C, Wu R-F, Huang Z, Chen Q (2019) Construction of a ceRNA network combining transcription factors in eutopic endometrial tissue of tubal factor infertility and endometriosis-related infertility. doi:10.21203/rs.2.19312/v1

58. Wong JC, Fiscus RR (2013) Protein Kinase G-I a Hyperactivation and VASP Phosphorylation in Promoting Ovarian Cancer Cell Migration and Platinum Resistance.

59. Kessler SM, Laggai S, Barghash A, Schultheiss CS, Lederer E, Artl M, Helms V, Haybaeck J, Kiemer AK (2015) IMP2/p62 induces genomic instability and an aggressive hepatocellular carcinoma phenotype. Cell Death \& Disease 6 (10):e1894

60. Reese KJ, Lin S, Verona RI, Schultz RM, Bartolomei MS (2007) Maintenance of paternal methylation and repression of the imprinted H19 gene requires MBD3. PLoS Genet 3 (8):e137

61. Zhao S, Bellone S, Lopez S, Thakral D, Schwab C, English DP, Black J, Cocco E, Choi J, Zammataro L (2016) Mutational landscape of uterine and ovarian carcinosarcomas implicates histone genes in epithelial-mesenchymal transition. Proc Natl Acad U S A 113 (43):12238-12243

62. Muro AF, Chauhan AK, Gajovic S, laconcig A, Porro F, Stanta G, Baralle FE (2003) Regulated splicing of the fibronectin EDA exon is essential for proper skin wound healing and normal lifespan. J Cell Biol 162 (1):149-160

63. Morley P, Armstrong DT, Gore-Langton RE (1987) Adhesion and differentiation of cultured rat granulosa cells: role of fibronectin. Am J Physiol 253 (5 Pt 1):C625-632. doi:10.1152/ajpcell.1987.253.5.C625

64. Asem EK, Carnegie JA, Tsang BK (1992) Fibronectin production by chicken granulosa cells in vitro: effect of follicular development. Acta Endocrinol (Copenh) 127 (5):466-470.

doi:10.1530/acta.0.1270466

65. Honda T, Fujiwara H, Yoshioka S, Yamada S, Nakayama T, Egawa M, Nishioka Y, Takahashi A, Fujii S (2004) Laminin and fibronectin concentrations of the follicular fluid correlate with granulosa cell luteinization and oocyte quality. Reprod Med Biol 3 (1):43-49. doi:10.1111/j.1447-0578.2004.00051.x

66. Ożegowska K, Brązert M, Ciesiółka S, Nawrocki MJ, Kranc W, Celichowski P, Jankowski M, Bryja A, Jeseta M, Antosik P, Bukowska D, Skowroński MT, Bruska M, Pawelczyk L, Zabel M, Nowicki M, Kempisty B (2019) Genes Involved in the Processes of Cell Proliferation, Migration, Adhesion, and Tissue Development as New Potential Markers of Porcine Granulosa Cellular Processes : A Microarray Approach. DNA Cell Biol 38 (6):549-560. doi:10.1089/dna.2018.4467 


\section{Table}

Table 1 General Characteristics of the patients with ovarian diseases

\begin{tabular}{|lllll|}
\hline Characteristics & $\begin{array}{l}\text { POl group } \\
(\mathrm{n}=10)\end{array}$ & $\begin{array}{l}\text { PCOS group } \\
(\mathrm{n}=10)\end{array}$ & $\begin{array}{l}\text { Control group } \\
(\mathrm{n}=10)\end{array}$ & $P$ value \\
\hline Age, years & $36.5 \pm 3.17$ & $30.2 \pm 3.62$ & $33.4 \pm 4.12$ & $\mathrm{~ns}$ \\
\hline $\mathrm{BMI}, \mathrm{kg} / \mathrm{m}^{2}$ & $21.86 \pm 2.29$ & $25.71 \pm 4.97$ & $23.22 \pm 3.00$ & $\mathrm{~ns}$ \\
\hline Basal FSH, IU/L & $29.45 \pm 4.79^{\mathrm{a}}$ & $5.32 \pm 1.18$ & $7.07 \pm 1.292$ & $<0.0001$ \\
\hline Basal LH, IU/L & $7.56 \pm 2.72^{\mathrm{a}}$ & $5.67 \pm 3.53$ & $3.56 \pm 1.37$ & $<0.01$ \\
\hline Basal $\mathrm{E}_{2}, \mathrm{pg} / \mathrm{mL}$ & $30.23 \pm 24.52$ & $36.68 \pm 7.67$ & $34.60 \pm 13.54$ & $\mathrm{~ns}$ \\
\hline Antral follicle number & $2.30 \pm 1.16^{\mathrm{a}}$ & $22.9 \pm 3.67^{\mathrm{b}}$ & $10.4 \pm 2.17$ & $<0.0001$ \\
\hline
\end{tabular}

Values are presented as mean \pm standard deviation.

Statistical differences were calculated using the non-parametric Kruskal-Wallis $\mathrm{H}$ test.

a Significantly different from POI group and Control group.

b Significantly different from PCOS group and Control group.

Abbreviations: $\mathrm{BMI}$, body mass index; $\mathrm{FSH}$, follicle-stimulating hormone; $\mathrm{LH}$, luteinizing hormone; $\mathrm{E}_{2}$, estradiol

Figures 


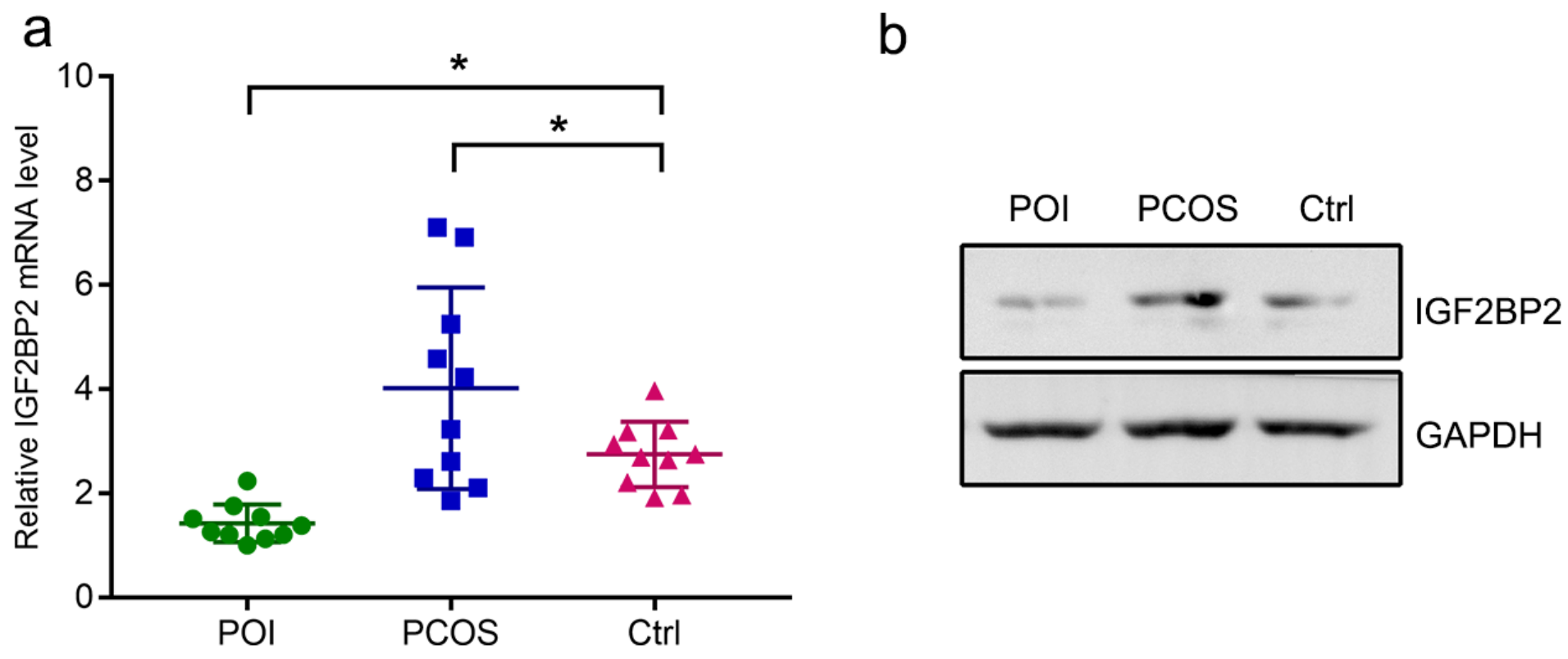

Figure 1

IGF2BP2 is differentially expressed in GCs from patients with ovarian diseases (a) IGF2BP2 mRNA detection by RT-qPCR in GCs from POI, PCOS and Comtrol women. (b) IGF2BP2 protein detection by western blot in GCs from POI, PCOS and Comtrol women. Data were normalized to glyceraldehyde 3phosphate dehydrogenase (GAPDH). Ctrl, control. Error bars represent the mean \pm SEM. ${ }^{*} p<0.05$.

a

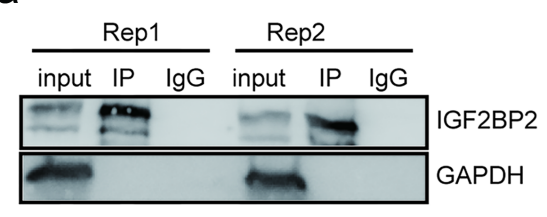

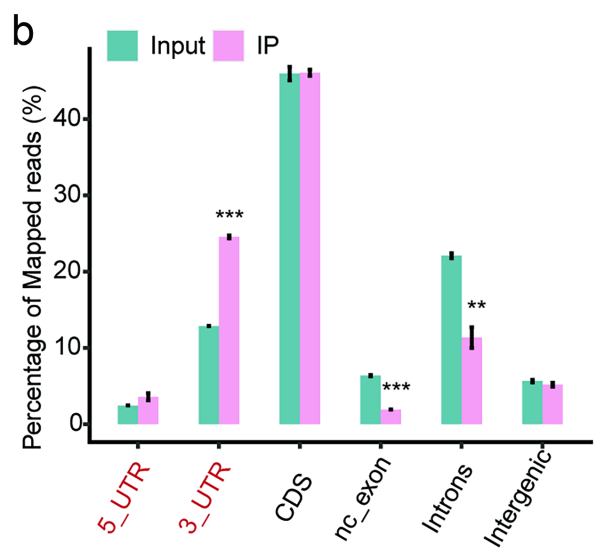

C

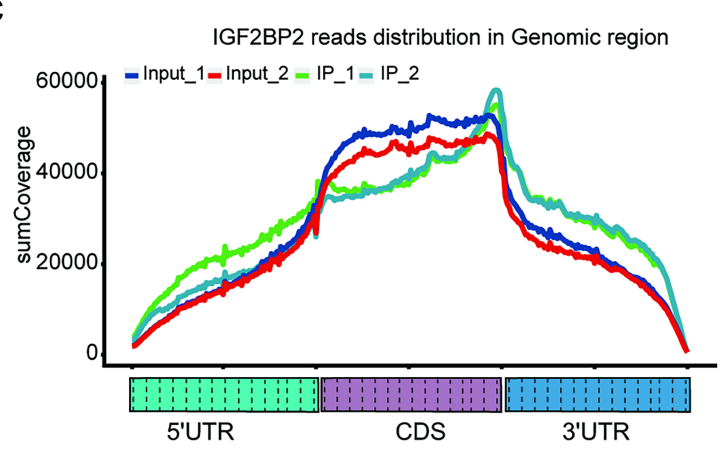

Figure 2

iRIP-seq analysis of IGF2BP2's binding profile in KGN cells (a) IGF2BP2 protein detection by western blot in KGN cells. (b) Read distribution across the reference genome. Error bars represent the mean $\pm \mathrm{SEM}$. **p $<0.01{ }^{*} \mathrm{p}<0.05$. (c) Peak read densities for 5'UTR, CDS and $3^{\prime}$ UTR for input and IGF2BP2 IP samples. 


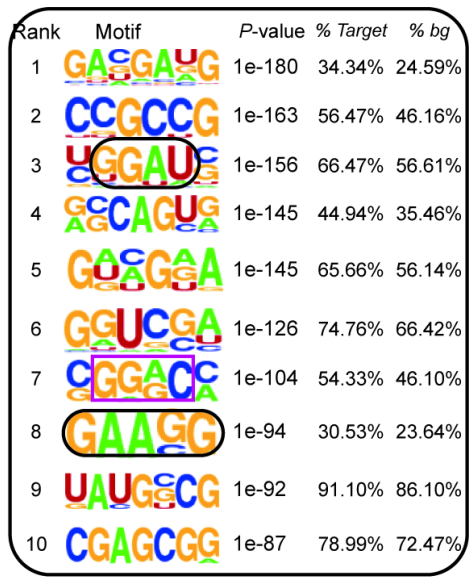

$b$



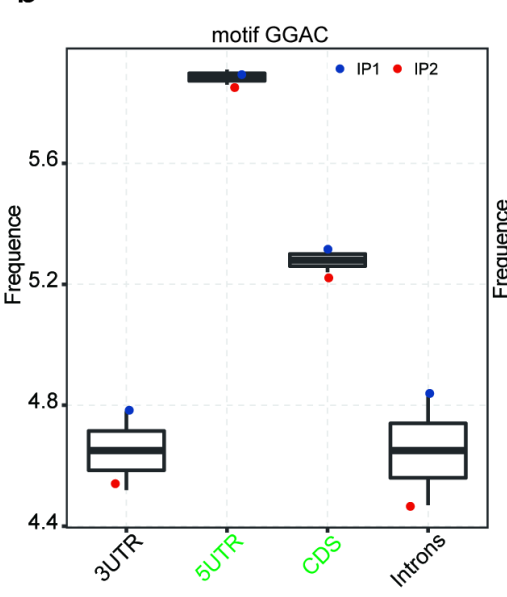

C



\section{Figure 3}

The binding motifs of IGF2BP2 and the binding sites (a) Motif analysis using the software HOMER showing the top 10 preferred binding motifs of IGF2BP2 (b) The binding sites of the GGAC binding motif. (c) The binding sites of the GAAG binding motif.



b IGF2BP2 IP olp GO enrichment $P$ top

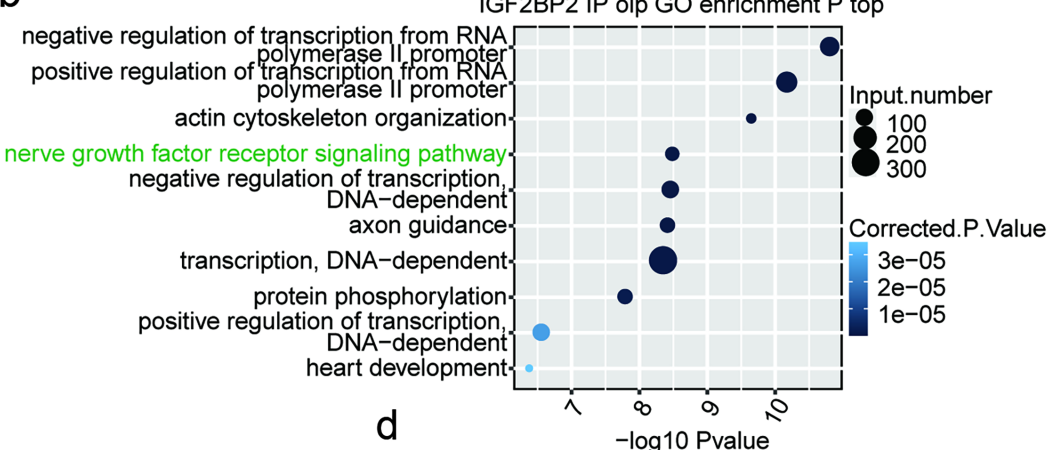

d

$-\log 10$ Pvalue



\section{Figure 4}

IGF2BP2-bound genes enriched in gene expression regulation (a) Venn diagram showing the overlap between peaks in two iRIP-seq sample replicates. (b) Bubble plot showing the top 10 biological processes associated with IGF2BP2-bound genes using a GO enrichment analysis. (c) Bubble plot showing the top 10 biological processes associated with IGF2BP2-bound genes using a KEGG enrichment analysis. (d) Bubble plot showing the top 10 pathways associated with IGF2BP2-bound genes using a reactome enrichment analysis. 
a

IP1 Peak _ IP2 Peak $\square$ IP1 \& IP2
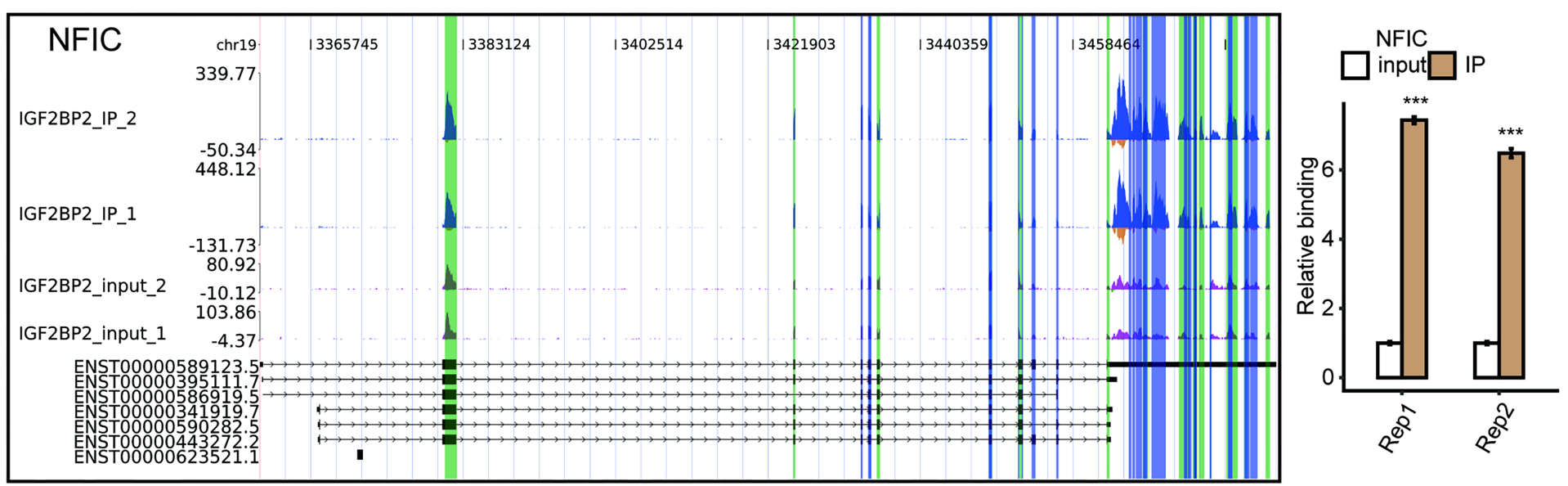

b
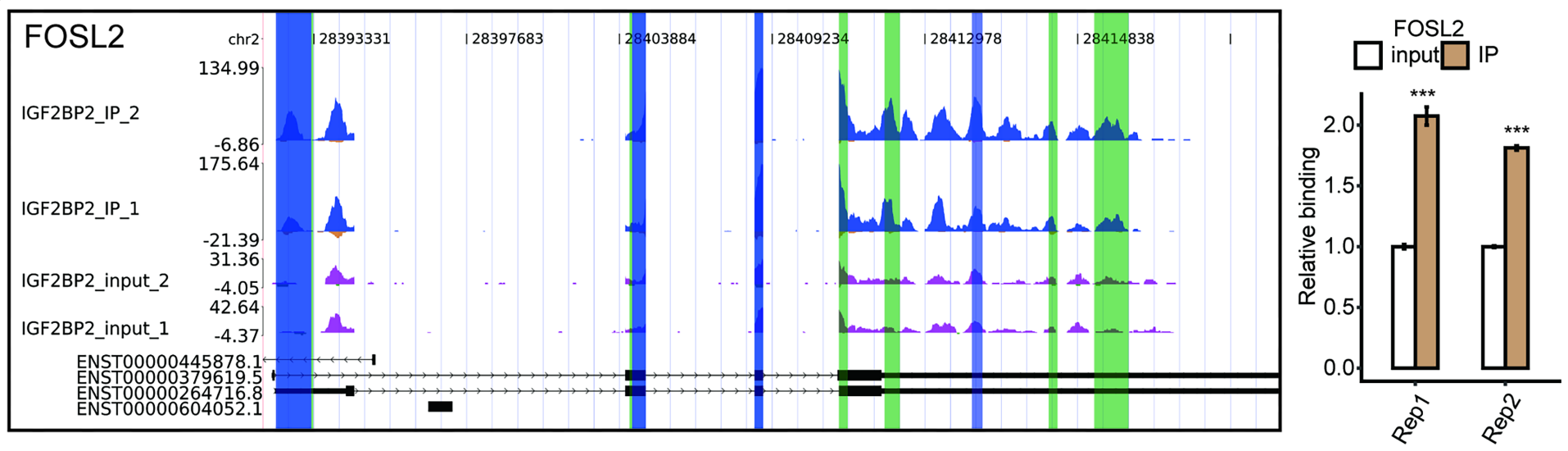

Figure 5

IGF2BP2 binds to the mRNAs of the genes NFIC(a) and FOSL2(b); genome visualization showing IGF2BP2-regulated ASEs and relative binding on the left and right panels, respectively. The peak ranges were highlighted with purple or green colors. 
a
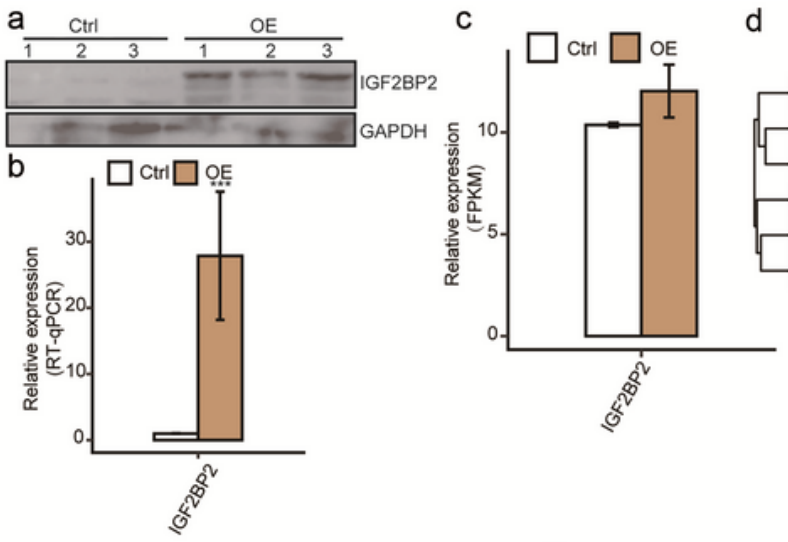

d
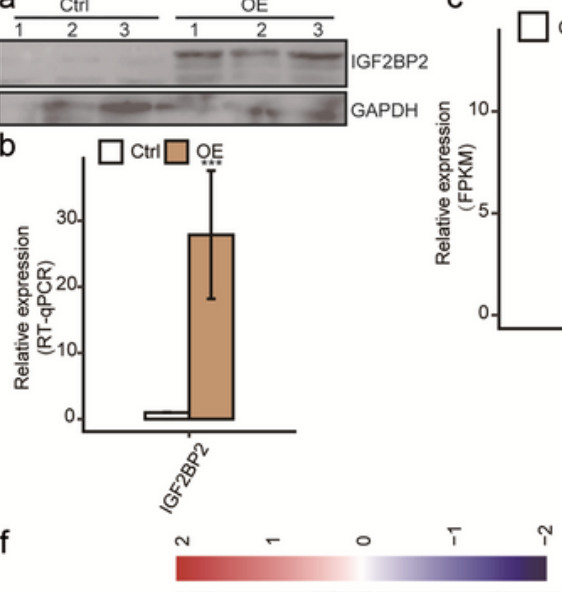

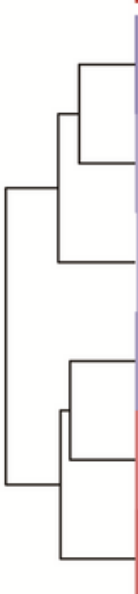



IGF2BP2 vs CtrI Down GO enrichment $P$ top

g

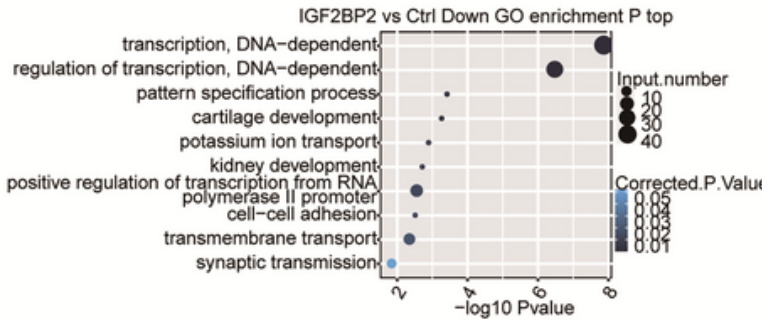

h
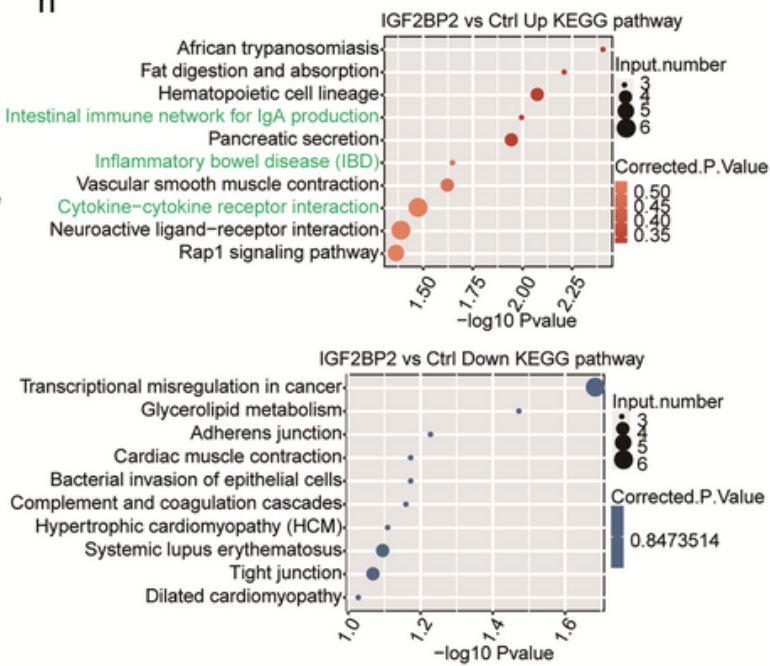

Figure 6

RNA-seq analysis of IGF2BP2 regulated transcriptome profile in KGN cells (a) IGF2BP2 protein detection by western blot. (b) IGF2BP2 expression levels quantified by RT-qPCR. Error bars represent the mean \pm SEM. ${ }^{* \star *}$ p $<0.001$. (c) IGF2BP2 expression quantified by RNA-seq data. FPKM values were calculated following the described in Materials and Methods. Error bars represent the mean \pm SEM. ${ }^{\star} p<0.05$. (d) Heat map showing a hierarchically clustered Pearson's correlation matrix resulting from the comparison 
between transcript expression levels for control and IGF2BP2-overexpressed samples. (e) Identification of IGF2BP2-regulated genes. Volcano plot shows up- and down-regulated genes labeled in red and blue, respectively. (f) Hierarchical clustering of DEGs in control and IGF2BP2-overexpressed samples. FPKM values were log2-transformed and then median-centered by each gene. $(\mathrm{g})$ The top $10 \mathrm{GO}$ biological processes of IGF2BP2 up- and down-regulated genes. (h) The top 10 KEGG pathways of IGF2BP2 up- and down-regulated genes.

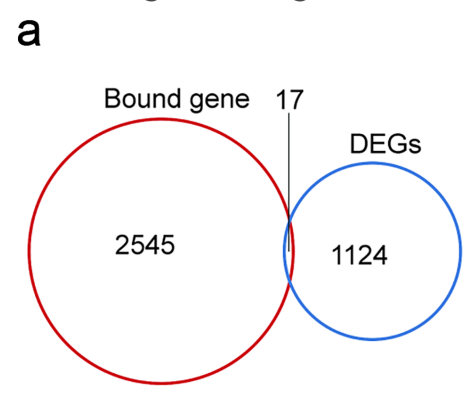

b $\square \mathrm{Ctr} \square \mathrm{OE}$
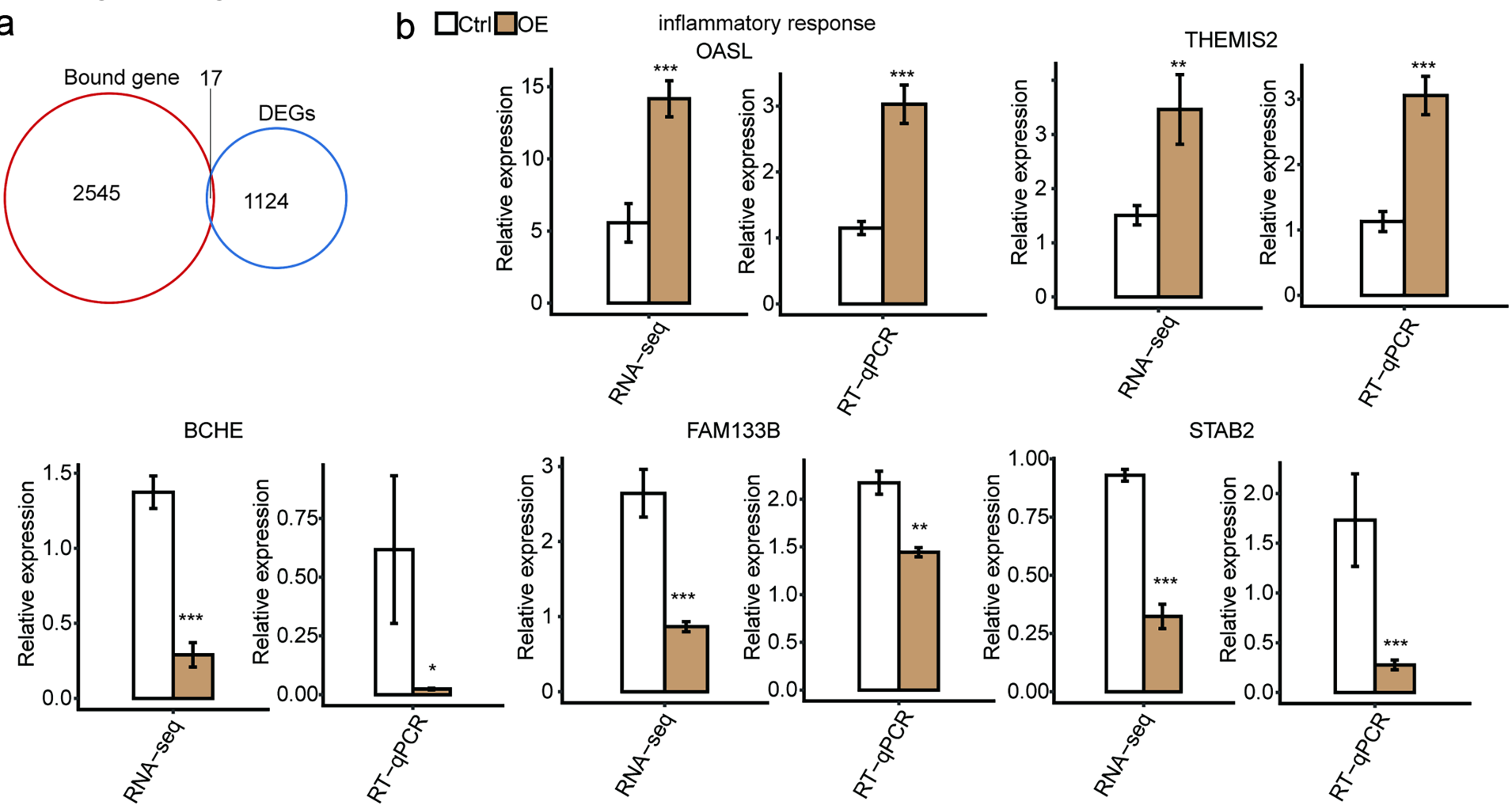

Figure 7

The DEGs regulated by IGF2BP2 overexpression (a) Genes overlapping between iRIP-seq and DEGs. (b) The relative expression of DEGs and $\mathrm{qPCR}$ validation. Error bars represent the mean $\pm \mathrm{SEM}$. ${ }^{* \star} \mathrm{p}<0.001$, $\star * p<0.01, * p<0.05$. 
a

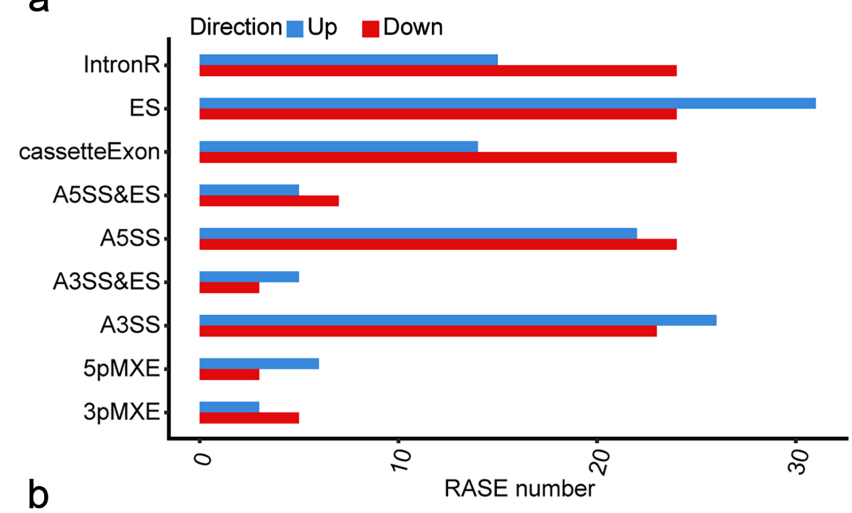

b

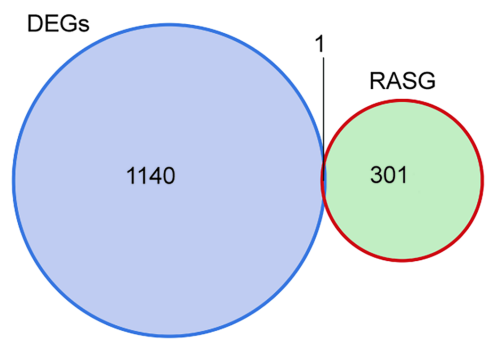

C

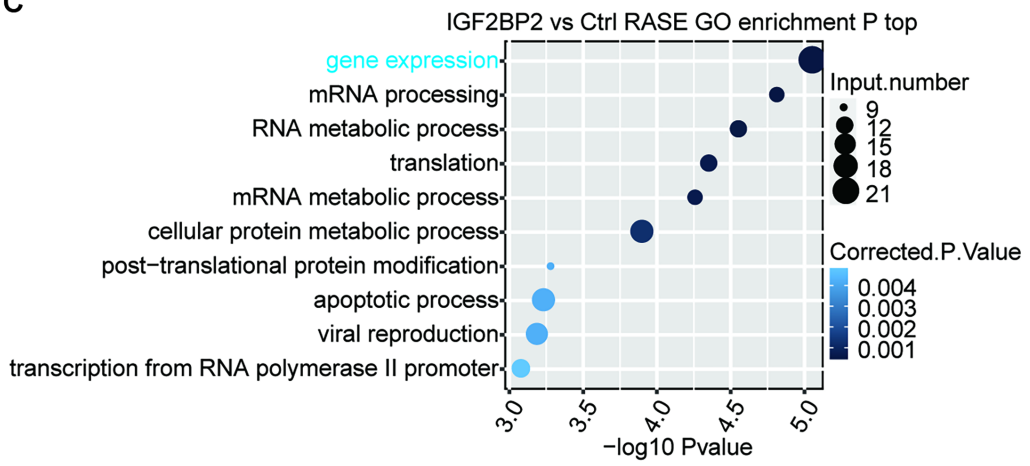

d

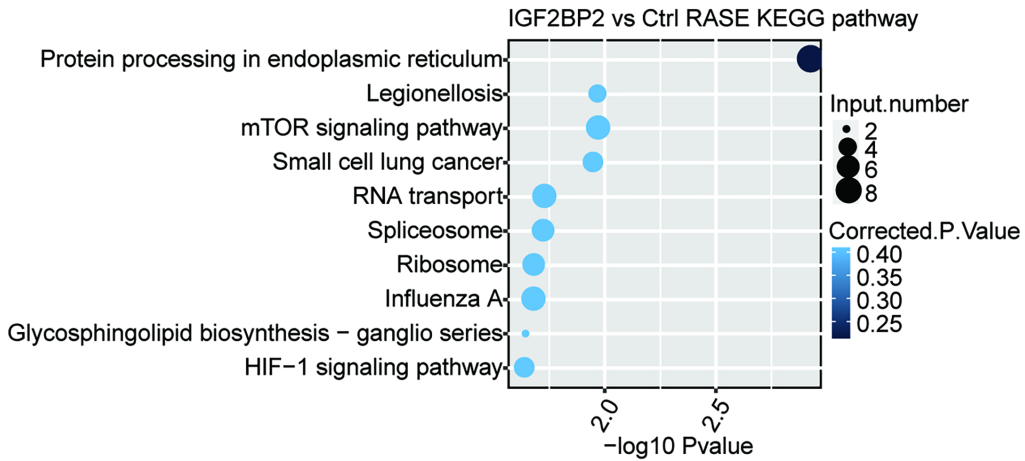

Figure 8

IGF2BP2-regulated alternative splicing events in KGN cells (a) Classification of IGF2BP2-regulated alternatively spliced events. (b) The overlap between IGF2BP2-regulated DEGs and RASGs. (c) The top 10 biological processes associated with IGF2BP2-regulated alternatively spliced genes using a GO enrichment analysis. (d) The top 10 pathways associated with IGF2BP2-regulated alternatively spliced genes using a KEGG analysis. 
a

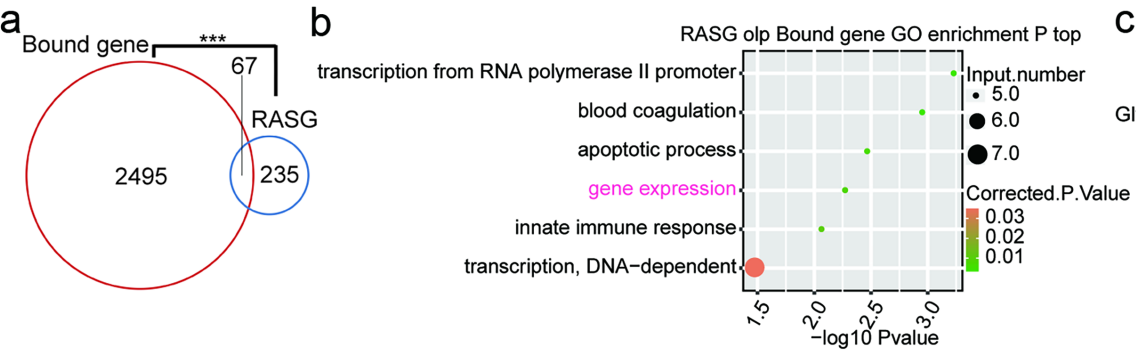

d

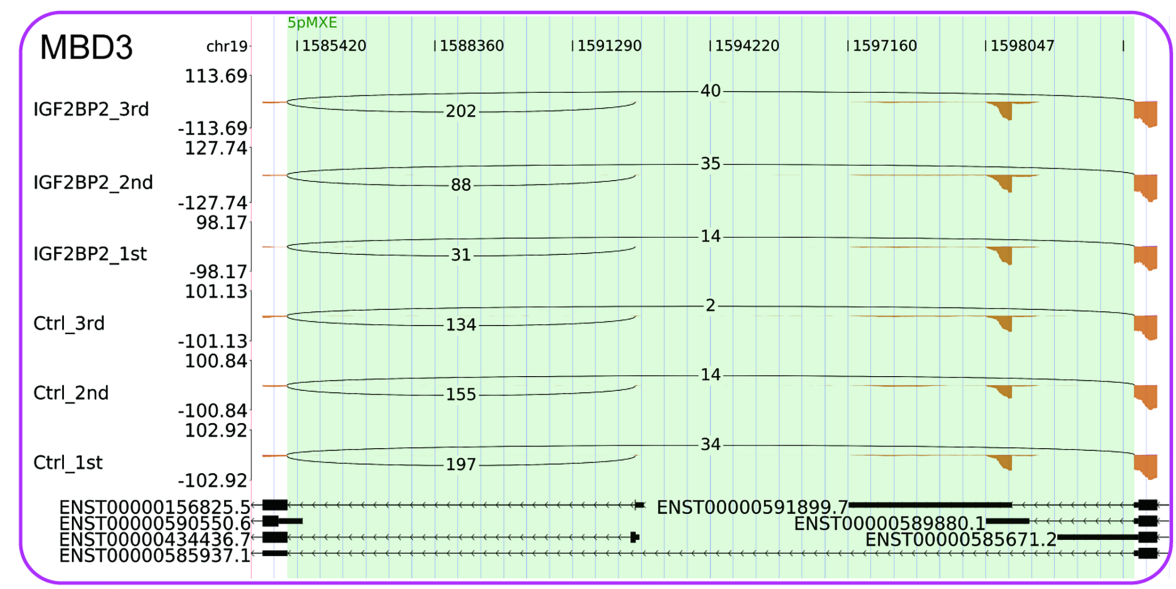

e

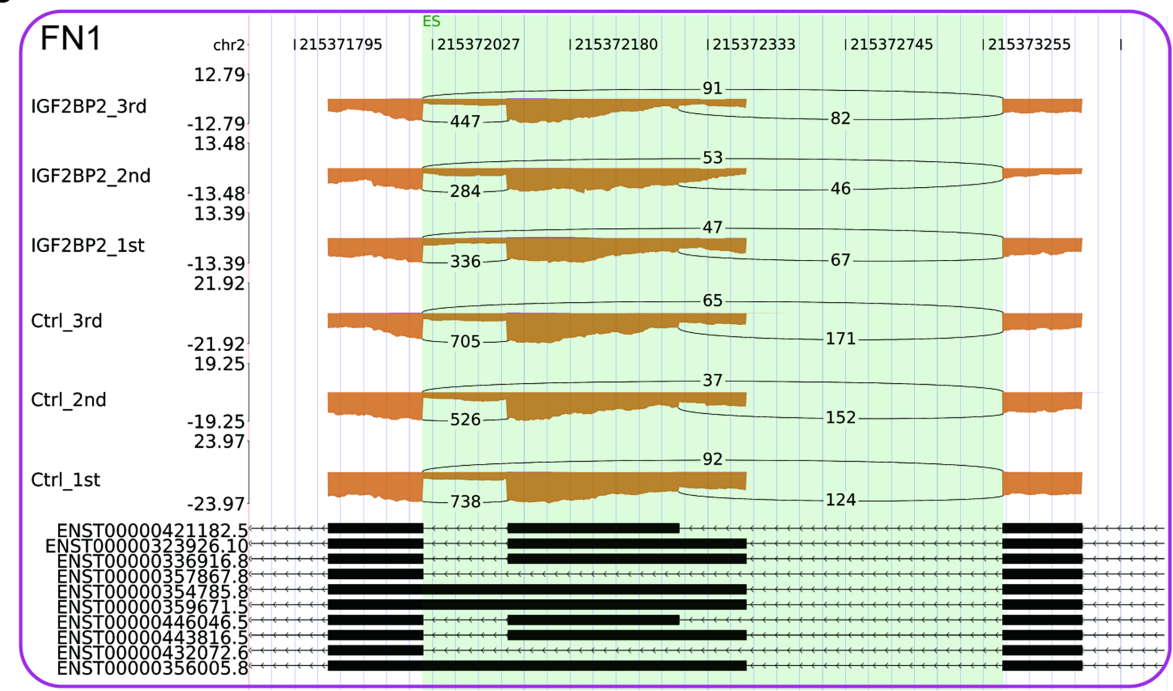

C
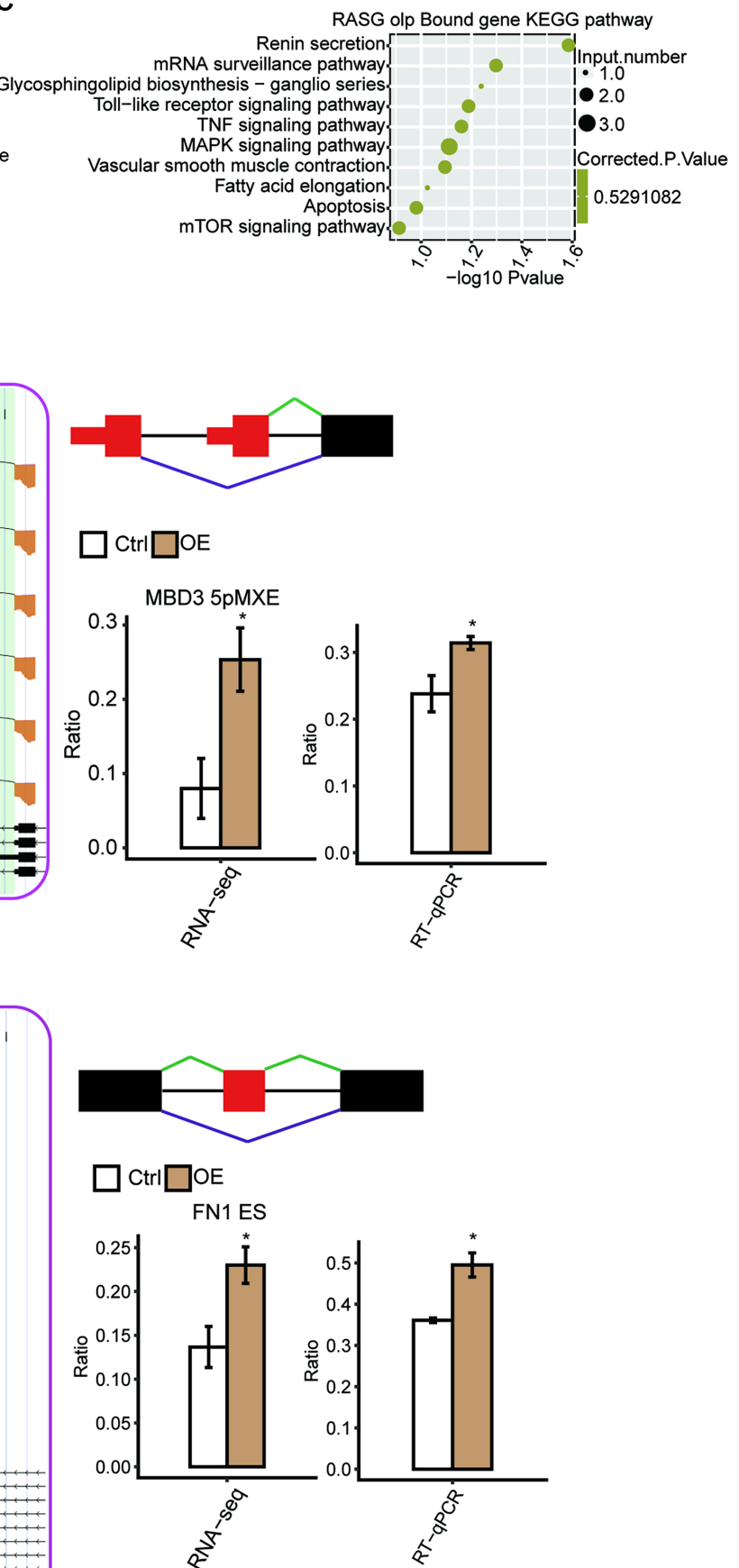

\section{Figure 9}

The alternatively spliced genes that bind to and are regulated by IGF2BP2 (a) Overlap between binding genes and RASG. (b) Bubble plot showing the biological processes of the genes overlapping as in (a) using a GO enrichment analysis. (c) Bubble plot showed the top KEGG pathways of the overlapped genes in (a). (d) IGF2BP2 regulates alternative splicing of MBD3, with IGV-sashimi plot showing an alternative $5 p M X E$ event. The altered ratios of ASEs in RNA-seq and in RT-qPCR are plotted on the right bottom panel. (e) IGF2BP2-regulated alternative splicing of FN1, with IGV-sashimi plot showing an exon skipping (ES) event. Read distributions of each ASE were plotted on the left panel with the transcripts of each gene shown below. The schematic diagrams depict ASEs structure, AS1 (purple line) and AS2 (green line). The 
constitutive exon sequences are denoted by the black boxes, intron sequences by the horizontal line (right panel, top), while the alternative exons are illustrated by the red boxes and the introns by the purple boxes. RNA-seq quantification and RT-qPCR validation of ASEs are shown at the bottom of the right panel. Error bars represent the mean \pm SEM. $* * *<0.001,{ }^{*} p<0.05$.

\section{Supplementary Files}

This is a list of supplementary files associated with this preprint. Click to download.

- Figs1.tif

- Figs2.tif

- SupplementaryTable120210225.xlsx 